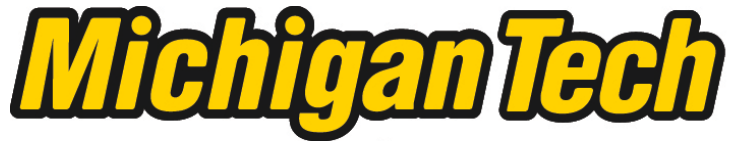 \\ Michigan Technological University Create the Future Digital Commons @ Michigan Tech
}

Dissertations, Master's Theses and Master's Reports - Open

Dissertations, Master's Theses and Master's

Reports

2015

STATE OF CHARGE BALANCING DROOP CONTROL

Rui Hu

Michigan Technological University

Follow this and additional works at: https://digitalcommons.mtu.edu/etds

Part of the Electrical and Computer Engineering Commons

Copyright 2015 Rui Hu

\section{Recommended Citation}

Hu, Rui, "STATE OF CHARGE BALANCING DROOP CONTROL", Master's Thesis, Michigan Technological University, 2015.

https://doi.org/10.37099/mtu.dc.etds/919

Follow this and additional works at: https://digitalcommons.mtu.edu/etds

Part of the Electrical and Computer Engineering Commons 


\title{
STATE OF CHARGE BALANCING DROOP CONTROL
}

\author{
By \\ R. $\mathrm{Hu}$
}

\begin{abstract}
A THESIS
Submitted in partial fulfillment of the requirements for the degree of MASTER OF SCIENCE

In Electrical Engineering

MICHIGAN TECHNOLOGICAL UNIVERSITY
\end{abstract}

2015

(C)2015 R. Hu 
This thesis has been approved in partial fulfillment of the requirements for the Degree of MASTER OF SCIENCE in Electrical Engineering.

Department of Electrical and Computer Engineering

\author{
Thesis Advisor : Wayne Weaver \\ Committee Member : Lucia Gauchia \\ Committee Member: Mahdi Shahbakhti \\ Department Chair: Daniel Fuhrmann
}




\section{Contents}

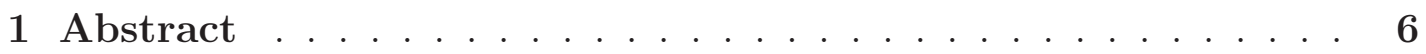

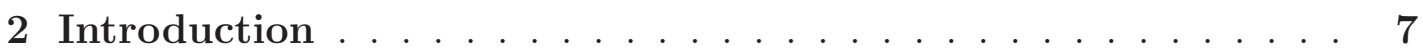

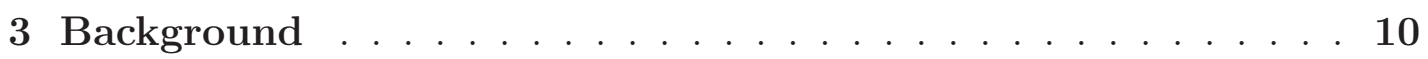

4 Proposed SoC Control Method . . . . . . . . . . . . . 17

4.1 Model of battery unit . . . . . . . . . . . . . . 17

4.2 Model of bi-directional converter . . . . . . . . . . . . . . . . 19

4.3 SoC control method . . . . . . . . . . . . . . . 20

4.4 SoC balancing speed . . . . . . . . . . . . . . . 26

4.5 Limitation on SoC droop Factor . . . . . . . . . . . . . . 27

4.6 Bus voltage-SoC detection . . . . . . . . . . . . . . . . . . . . . 29

4.7 Method summary . . . . . . . . . . . . . . . . . 30

5 Simulation Study . . . . . . . . . . . . . . . 31

5.1 Simulation of two batteries support one load . . . . . . . . . 31

5.2 Simulation of batteries with different charging coefficient . . . . . 34

5.2.1 Impact of difference in battery charging coefficient . . . . . . 34

5.2.2 Droop factor compensation for battery discharging coefficient difference . . . . . . . . . . . . . . . . . 36

5.3 System with power source $\ldots \ldots \ldots \ldots$

5.4 Simulation on charging/discharging switch process . . . . . . . 41

5.5 Application of $V_{b u s}-S o C$ detection $\ldots \ldots \ldots \ldots . \ldots 42$

5.6 Simulation summary . . . . . . . . . . . . . . . . . . . . 47

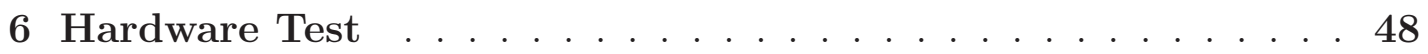


6.1 Scenario I: Two identical batteries support one load . . . . . . . . . 49

6.2 Scenario II: Two batteries support one load with different discharging coefficient .................. . . 54

6.3 experiment conclusion .................. . . 54

7 Conclusion and future work . . . . . . . . . . . . . 58

8 References ......................... 60

9 Appendix ......................... 65

9.1 Simulation block scheme in MATLAB/Sinmulink . . . . . . . . 65

9.2 Control model for electronic drive board in Sinmulink . . . . . . . . 68

9.3 Hardware test material . . . . . . . . . . . . . . . . . 69 


\section{List of variables}

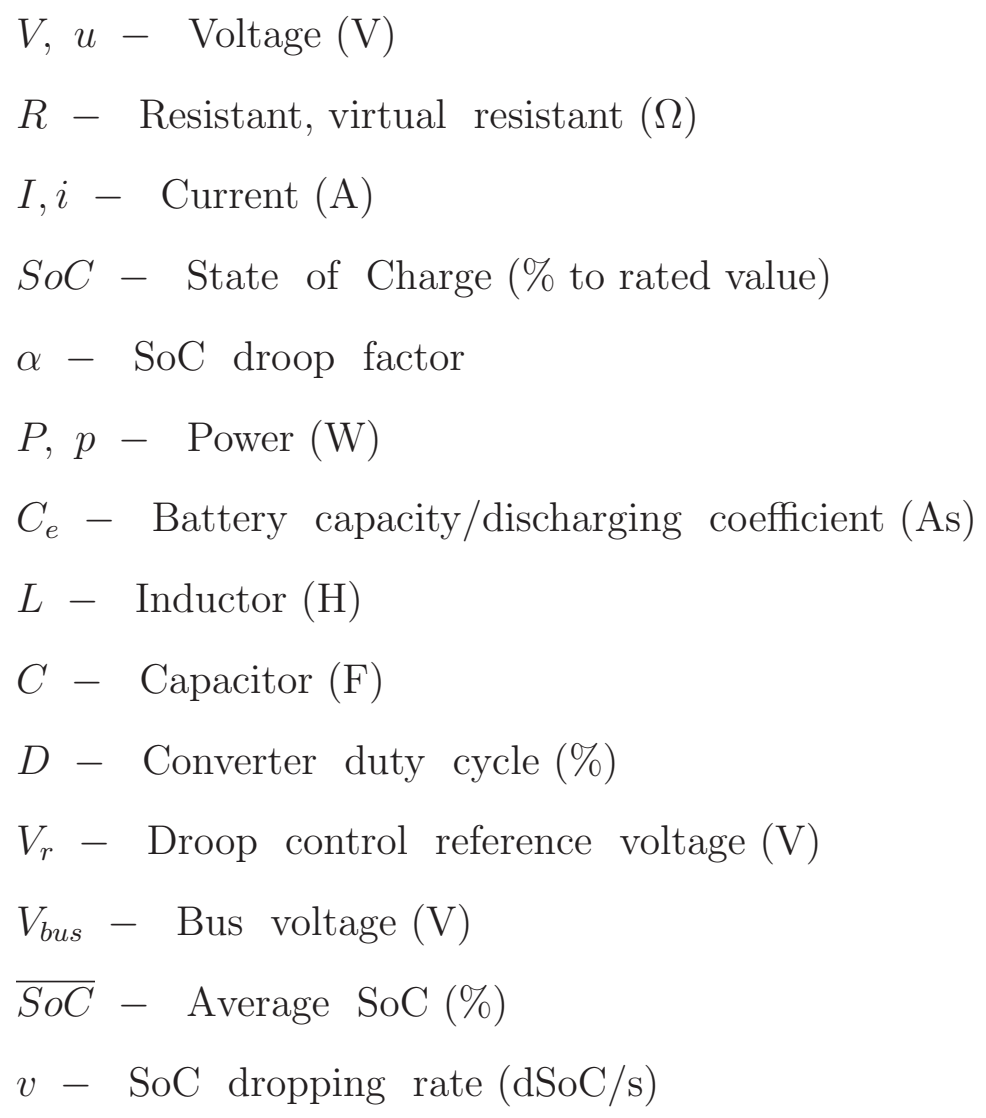




\section{Abstract}

This thesis presents a load sharing method applied in a distributed micro grid system. The goal of this method is to balance the state-of-charge (SoC) of each parallel connected battery and make it possible to detect the average SoC of the system by measuring bus voltage for all connected modules. In this method the reference voltage for each battery converter is adjusted by adding a proportional SoC factor. Under such setting the battery with a higher SoC will output more power, whereas the one with lower SoC gives out less. Therefore the higher SoC battery will use its energy faster than the lower ones, and eventually the SoC and output power of each battery will converge. And because the reference voltage is related to SoC status, the information of the average $\mathrm{SoC}$ in this system could be shared for all modules by measuring bus voltage. The SoC balancing speed is related to the SoC droop factors. This SoC-based load sharing control system is analyzed in feasibility and stability. Simulations in MATLAB/Simulink are presented, which indicate that this control scheme could balance the battery SoCs as predicted. The observation of SoC sharing through bus voltage was validated in both software simulation and hardware experiments. It could be of use to non-communicated distributed power system in load shedding and power planning. 


\section{Introduction}

Microgrid and smart grid have become a swiftly developing study area through the last decade. Due to a vast improvement of power electronics devices and their application, de micro grid now has earned its positions in many industrial systems and applications. Among them, distributed energy resource (DER) is one of a kind, it is becoming more and more popular in many industries. Distributed power scheme offers considerable advantages than traditional power system structure, which includes: enhanced system stability, high energy efficiency in low voltage levels, lower maintenance cost and modularity [1].

One problem distributed power has to deal with is load sharing, when multiple sources are connected in one bus parallely, they are expected to share the load in some way. For a system with energy storage devices, it is required that the energy storage devices absorb and output uniform power [2]. One solution for this problem is a central controller from which all converters receive control signals. The central controller gathers information through an over-all communication system from each device, and sends out proper controlling signals based on its embedding algorithm [3]. An alternative way is to have decentralized controllers implemented in each power source and load, which uses their own sensor, communication network, processor with corresponding algorithm. A widely known algorithm for decentralized controller is droop control, which

has been intensively studied in the past decades $[4,5,6]$. A droop controller regulates its converter reference output current based on a dropping voltage-current curve, when the output power increases the bus voltage would drop, where the bus voltage depends on load conditions.

There are many different ways in realizing droop control, most of them do 
not take storage device status into consideration. But in some applications the storage device status could be an important issue. For hybrid electric vehicles (HEV), the priority of controlling system is to obtain close to $100 \%$ charge efficiency or charge acceptance [7], while the charge acceptance for a battery varies with its SoC. At a low state of charge the charge acceptance is close to $100 \%$ and becomes progressively poorer when above $80 \%$ SoC, thus the controller should try to maintain SoC to a certain low value as close as possible [8]. Under such condition, detection and communication of battery SoCs values become vital important. In [9] a set of accurate SoC sensor and monitor system was attached to regulate batteries' output power. But such controlling scheme may lead to conflict of interest with the micro power system, especially when droop control method is applied. Droop control demands each power source to deliver power depending on load condition and their default droop factor rather than battery states, without proper setting the two control systems could end up fighting with each other and causes stability issue. To over come this, droop controller should take the battery status into consideration.

In this thesis a modification factor was added into droop method based on SoC balancing. The mathematical analysis and simulation result show that such setting could balance the SoC of batteries effectively. And due to the SoC-V feature of this control method, that the each converter reference voltage was set based on SoC state, the whole system SoC states could be shared by observing bus voltage among batteries, loads or other sources.

This thesis consists of several parts: background, simulation study and hardware test. Chapter 3 will provide a detailed background on the use of conventional droop control, existing SoC balancing droop control and the presented one. There will be a brief compare between different methods in droop curves. 
In chapter 4 , the controlling method used in the SoC balancing droop control and its features will be described and discussed. The model applied in the mathematical analysis will be explained as well. Chapter 5 will focus on the simulation of this control method in different applications, which includes battery charging and discharging process and different battery coefficients' impact to the balancing effect. Chapter 6 will show a hardware test validating this method. In the hardware test a system with two power sources supporting one load using presented method will be presented. Analysis of the sensed data proved that this method could achieve its object. All results show that this method could successfully balance the SoCs of batteries in a system and put the average SoC information on the bus line. 


\section{Background}

Most SoC balancing studies focused on the series linked battery unit current sharing problem $[10,11,12]$, because battery unit was usually treated as a single grouped module in the system. In papers studying this kind of problem, the battery power sources would be classified as a single battery unit or a series connected battery bank. But in some applications, especially in distributed power systems, each user, load or power source could have its own energy storage device. They could be flywheel, fuel battery, traditional battery or storage capacitors. In this kind of system the load sharing is not just about the series battery current arrangement issue but a parallel load allocation problem.

To solve this battery load sharing problem, the distributed energy storage units (ESUs) are commonly applied in a micro grid [13]. Usually an ESU consists of two parts: a battery management system(BMS) and a power converter system(PCS). The main function of BMS is to balance the string batteries' SoCs and control their output voltage, which has been mentioned. The power converter system(PCS) is in charge of controlling the converters and regulating their output currents, voltages and power. It is possible to solve the parallel SoC balancing among battery units via controlling the PCS. In [14] a cascade H-bridges is employed in PCS to balance the SoC of each ESUs, while in [15] a low-pass filter is applied in the control system to improve the load distribution, which consists of several kinds of ESUs. But all the methods mentioned above were revealed by using a central control system, which is not very suitable for decentralized micro systems.

Droop control method has been applied in a wide array of control systems. In electrical systems it could be used in many scenarios like bus voltage regulating, 
automatic generation control (AGC) and motor control. For a DC grid droop scheme, it could be simply described as decreasing the output voltage when the output current increases. One conventional droop control application is to program the converter controller to regulate its output impedance to achieve current and power sharing among power supply sources. One feature of this method is that with better current sharing, worse voltage regulation occurs. According to [16], two advantages of droop control versus other load sharing methods are that: it needs no wire connections among control circuit of parallel converters and its simplicity in implementation and expansion. The controller will sense the value of output current and voltage of each power converter, and the reference voltage equation it would use is

$$
V_{i}=V_{\text {set }}-R_{\text {droop }} I_{i},
$$

where $V_{i}$ is the $i_{t h}$ converter output port voltage, $V_{\text {set }}$ is open circuit reference voltage, $R_{\text {droop }}$ is droop factor and $I_{i}$ is the $i_{t h}$ converter output current.

Fig.1 shows how droop control works. For two parallely connected converters, their droop curves are shown as in Fig.1. The converters try to regulate their current based on the equation $V=V_{\text {ref }}-R I$. Since they are parallely connected their output voltages are nearly the same. Assuming bus voltage is $V_{\text {bus }}$, the two converters would locate their own operating point on the curve with the same voltage value. For converter 1 its desired current is $I_{1}$, therefore it should regulate its current to $I_{1}$ under such settings and so does converter 2. Therefore the load sharing object is fulfilled. This process is shown in a simulation concerning two power sources supporting one load. A diagram of this simulation is shown in Fig.2. In this simulation, converter 2 has a larger droop factor, so its desired 


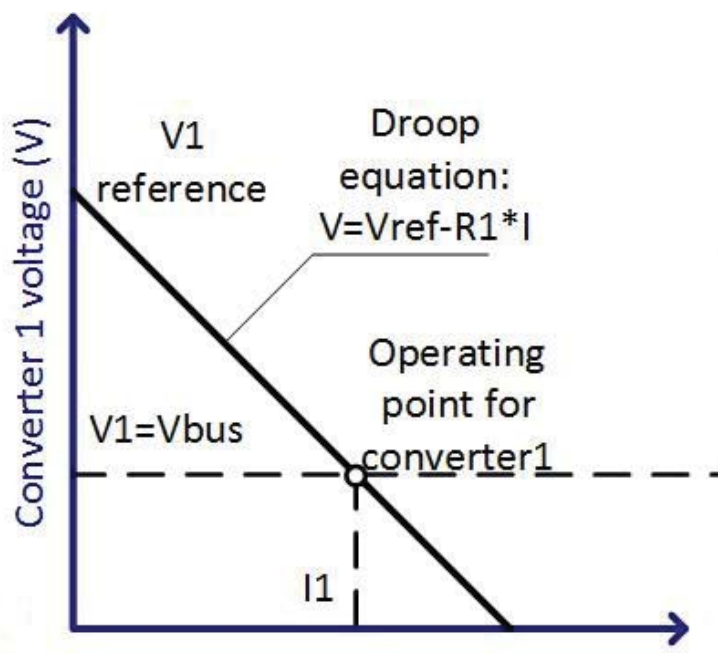

Converter 1 current(A)

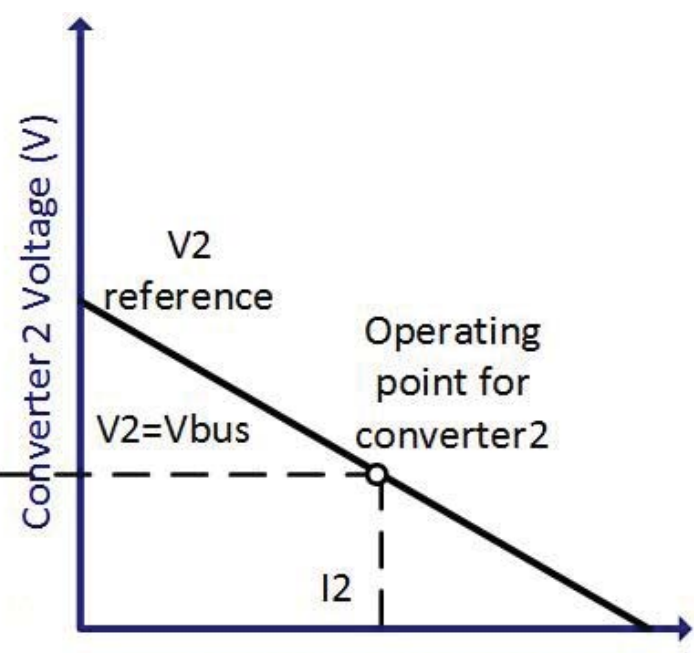

Converter 2 current(A)

Figure 1: Droop control principle shown in V-I curves

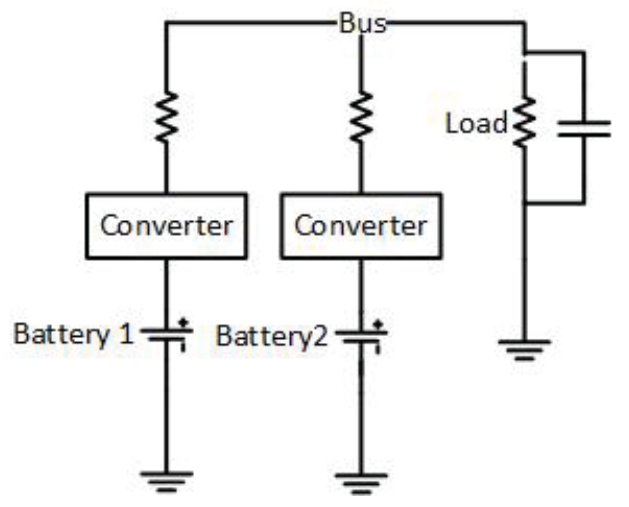

Figure 2: Simulated system applying droop control

output current is smaller, which can be seen from the simulation currents' output in Fig.3. In the simulation the load had a step change at 25 second, and the converter output currents were reallocated after that, revealing that the current sharing was affected by load condition too.

Each converter programmed in this method would regulate its output power according to bus voltage and its droop factor $R_{\text {droop }}$. As mentioned in the introduction, conventional droop control focuses more on load sharing and tracking 


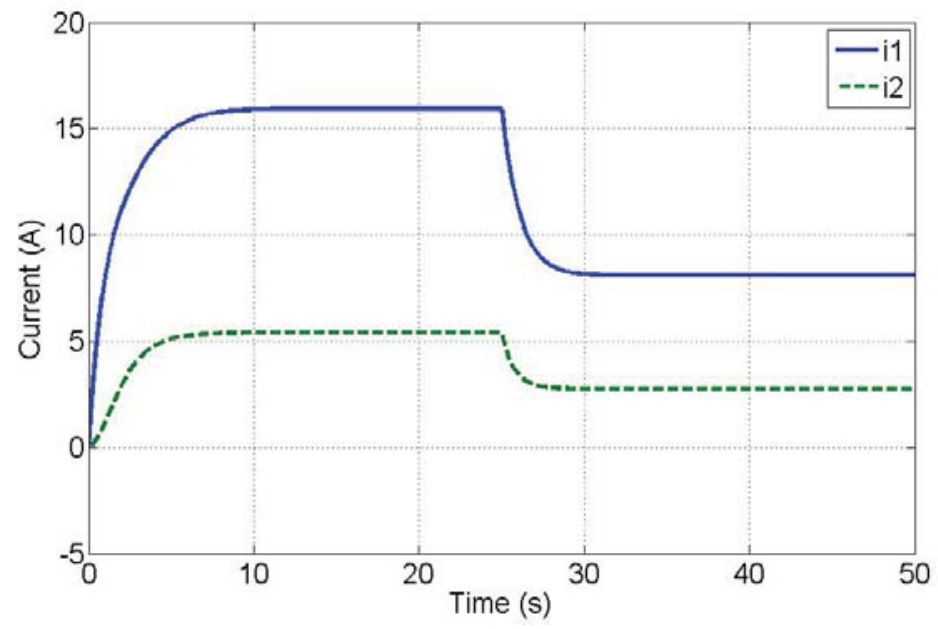

Figure 3: Droop control simulation, current of converters, current were reallocated at $25 \mathrm{~s}$, when a load step change occurred

speed, while in some applications the storage device status is no less important. In a system with energy storage devices, the storage devices' capacity, output power limitation and device charging/discharging status are all vitally important to the health of the storage devices as well as operation of the system. For example, requirement of the battery's operating $\mathrm{SoC}$ is different. Usually it is a required range for SoC. To achieve that, the converters of sources and loads have to get the information about the SoC status of batteries in the system and make adjustment accordingly. Therefore each battery's SoC value has to be sensed and sent to each converter via communication lines. Such modification is straightforward but the advantages of droop control have been erased entirely: it needs both a communication network now and more work in the building process. The proposed solution is to combine the local SoC status with the droop control scheme, but still, without communication each converter cannot get information about other batteries' SoC status. One way to solve this is to balance SoCs of all batteries in the system and share it in power lines. 
Among all different methods realizing droop control, there are two papers presenting parallel SoC balancing methods. Their ideas are similar; their droop factor from $[17,18]$ have reference voltage equations as

$$
V_{i}=V_{\text {set }}-\frac{a_{\text {droop }}}{S o C^{n}} p_{i}
$$

where $S o C$ is the battery state of charge, and $p_{i}$ is the converter output power.

The purpose of this method is to let the storage device with a higher SoC output more power than others, to do that the high SoC battery would regulate its power based on a smaller droop factor $a_{d r o o p}$, which would lead to a higher reference current $I_{i}$. The tracking speed of the SoC of this method could be modified through adjusting the power factor $n$. This is the discharging strategy of the control method when the system has to use batteries to meet the load demand. And when the power supply in the system is sufficient, each battery would be controlled to get its maximum charging current/power in order to get fully charged as fast as possible.

The main principle of this method is shown in Fig.4. Assume the converter was connected to a battery with $50 \%$ SoC while converter 2 was connected to a fully charged battery. Set $\mathrm{n}$ to 1 , from the droop relationship $V_{i}=V_{\text {set }}-\frac{a_{\text {droop }}}{\operatorname{SoC}^{n}} p_{i}$ the converter 1 droop curve would become the dotted one in Fig.4 with a steeper drooping rate. From Fig.4 it can be told that converter 1 would output less current than in the former situation and therefore its SoC would drop slower too.

Theoretically during the charging process, the SoC-adjusting method could be applied as well. Power that exceeds the need of the load might not be sufficient to charge all batteries at the same time, and in order to balance the SoC the 

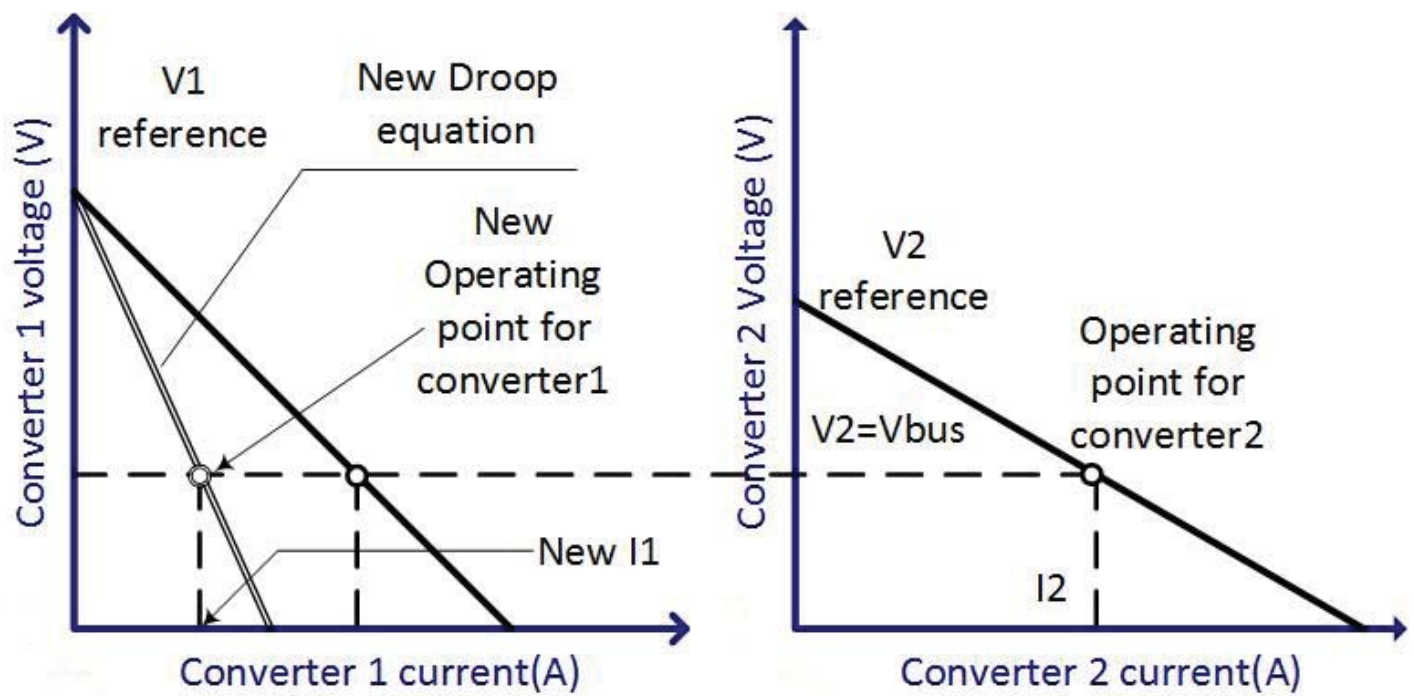

Figure 4: SoC Droop control principle 1

batteries with lower SoC should be charged more.

The method presented in this thesis is similar to the one mentioned above, in that they both try to modify the droop equation based on the SoC status of batteries. In this thesis the method modifies the droop reference voltage value instead of the droop factor. The droop equation is $V=V_{\text {ref }}+\alpha S o C-R I$. This process was depicted in Fig.5. Considering the same condition as shown before, converter 1 has a half charged battery. The converter 1 reference voltage would be $V_{\text {ref }}+0.5 \alpha$ while converter 2 reference voltage be $V_{\text {ref }}+\alpha$. The droop curves for converters would become those new two in Fig.5 (in this scenario the initial voltage references have been modified to have $\left.V_{\text {newref }}+75 \% \alpha=V_{\text {ref }}\right)$. Then converter 1 would reduce its current while converter 2 would increase, and the batteries SoCs could be balanced as well.

Additionally, in this method one feature was-by the application of the SoCbalance droop method- that the SoC information could be shared in the system via bus voltage directly without communication lines. This could be an advan- 


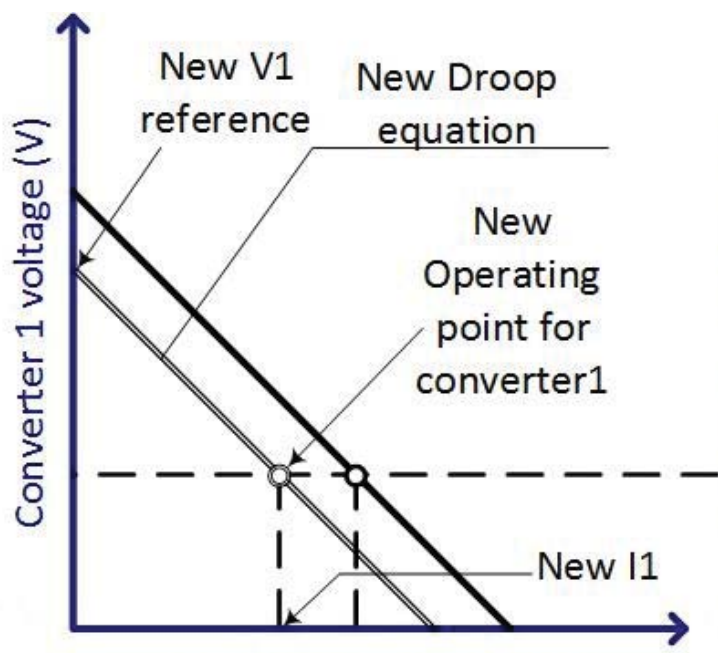

Converter 1 current(A)

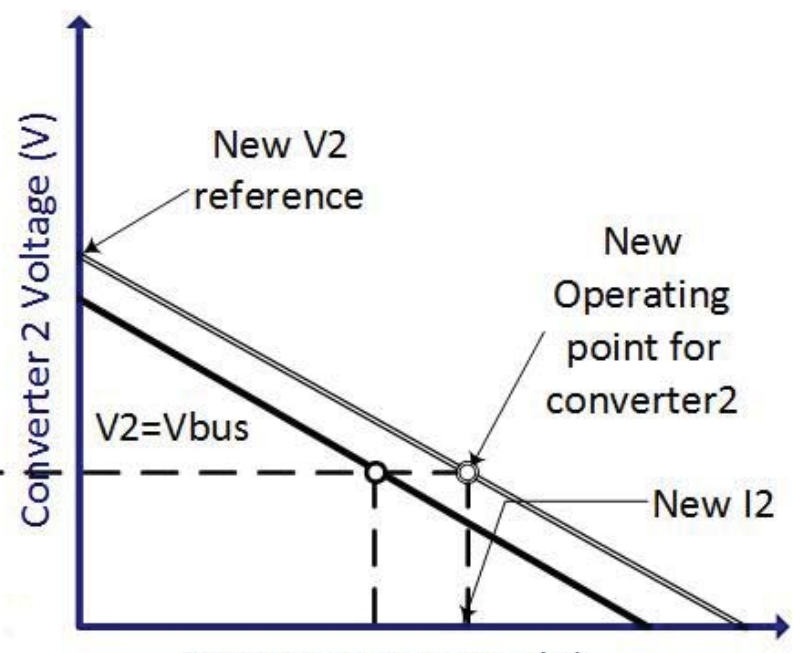

Converter 2 current(A)

Figure 5: SoC Droop control principle 2

tage for some separated micro grid with individual loads, storage devices and sources, for which a communication line is costy.

In this chapter, principles of conventional droop control, existing and proposed SoC balancing method were demonstrated and compared. Conventional droop control could accomplish the load sharing object decently, but in some applications of microgrid, the energy storage devices status should also be taken its consideration. Existing SoC balancing method modifies the droop equation by adjusting the droop factor based on battery SoC states, while the presented method changes the droop reference voltage according to SoC status. Because of the relation between SoC and converter voltage built using presented method, and because that all battery SoCs are balanced, the SoC information could be shared among all devices connected to the system by measuring the bus voltage. 


\section{Proposed SoC Control Method}

In this section the SoC control method, mathematical model and the parameter settings of modules are presented. Steady state and state-space equations describing the system are shown. Based on them a stability analysis is done, the results showed that the studied system is stable using this control strategy. One feature of this method is that the difference in battery capacity/charging/discharging coefficients could affect the SoC balancing. After that, the formula of SoC balancing speed is given, its features are discussed, revealing that a large SoC droop factor could increase the tracking speed. The SoC droop factor's several practice application limitations, both on system voltage and batteries requirements, are listed. To fulfill these requirements, the SoC droop factor has to be set under corresponding restrictions. Lastly the voltageSoC detection feature is presented, showing that all converter controllers could get the information of system average SoC value by measuring the bus voltage.

\subsection{Model of battery unit}

Since the battery model used in this system only needs to demonstrate specific feature, which is the relation between SoC and output voltage, there is no need in building an over detailed dynamic battery model covering charging/discharging process. Therefore the battery model used in this thesis is a simplified controlled voltage source, whose Voltage-SoC curve is shown in Fig. 6. The equation form is built based on [17], parameters of battery have been modified to acquire sufficient output power.

The curve fitting tool in MATLAB was used to find an approximation SoC-V function of this battery. The fitting algorithm is trust-region, and a two terms 


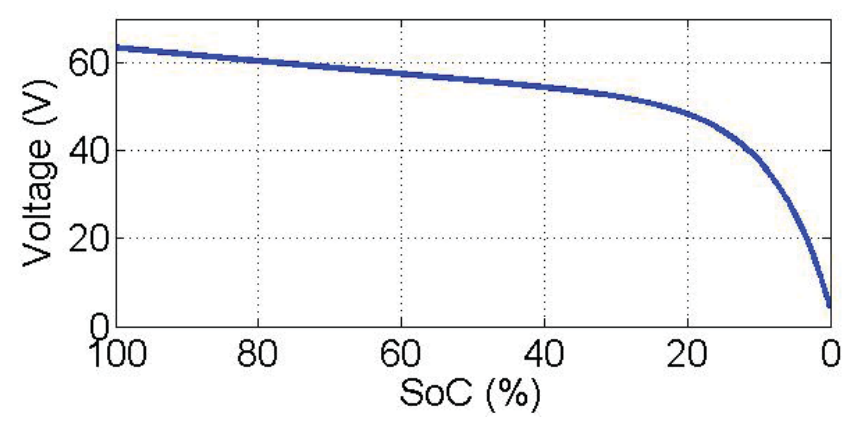

Figure 6: Battery SoC-Voltage fitting

exponential $y=a \exp (b x)+c \exp (d x)$ (a, b, c, d are undetermined coefficients) was chosen to be the fitting function. In the end the battery model Voltage-SoC equation was given as

$$
V_{\text {battery }}=49.76 e^{(0.2454 S o C)}-45.17 e^{(-12.35 S o C)}
$$

where $V_{\text {battery }}$ is the battery output voltage, $e$ is Euler's constant. The SoC value in this model is calculated from

$$
S o C(t)=S o C_{0}-\frac{1}{C_{e}} \int i_{\text {out }} d t
$$

where $S o C(t)$ is the current $\mathrm{SoC}$ value, $S o C(0)$ is the initial SoC value, $C_{e}$ is the capacity of the battery, and $i_{\text {out }}$ is the battery output current. The SoC value was sent to the controller. In this thesis the battery capacity $C_{e}$ will be called as the charging and discharging coefficient. 


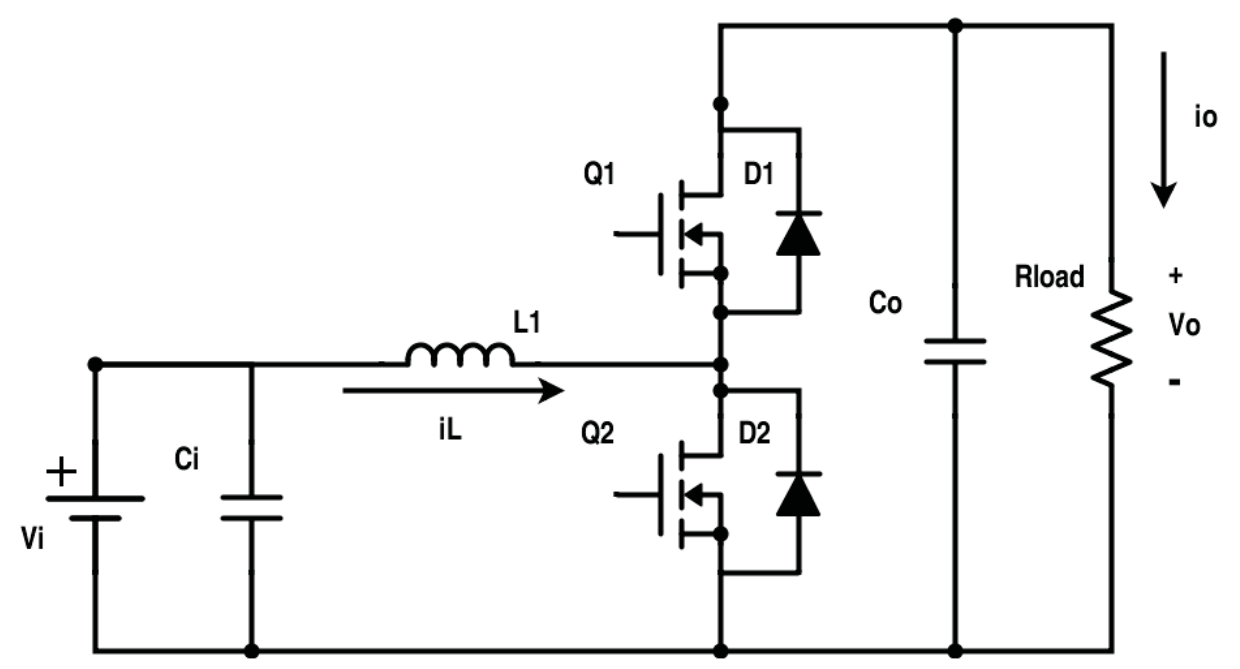

Figure 7: Bi-directional DC/DC converter model

\subsection{Model of bi-directional converter}

Converter used in the simulation is a bi-directional boost/buck DC/DC switching converter, whose output side voltage would always be higher than its input. But the current could flow form the lower voltage side to the higher or inverse. Scheme of this converter is shown in Fig. 7, this model is built using differential equations without switching part in pulse width modulation(PWM). The equations describing this converter are

$$
\begin{aligned}
L \frac{d i_{L}}{d t} & =V_{i}-D V_{o} \\
C_{o} \frac{d V_{o}}{d t} & =i_{o}-D i_{L}
\end{aligned}
$$

where $L$ is the inductor, $C_{i}$ is the input capacitor, $C_{i}$ is output capacitor, $V_{i}$ and $V_{o}$ are the input and output voltage, $i_{L}$ is the inductor current, $i_{o}$ is output current, and $D$ is the duty cycle for switches.

In the system that will be introduced in the next section, the output voltage 
would be regulated to a certain value, that $\frac{d V_{o}}{d t} \rightarrow 0$ and $I_{o}$ is a fixed value. From (6) it can be deduced that $i_{L}=D i_{o}$, meaning that the converter could regulate the current flow in and out of the source.

\subsection{SoC control method}

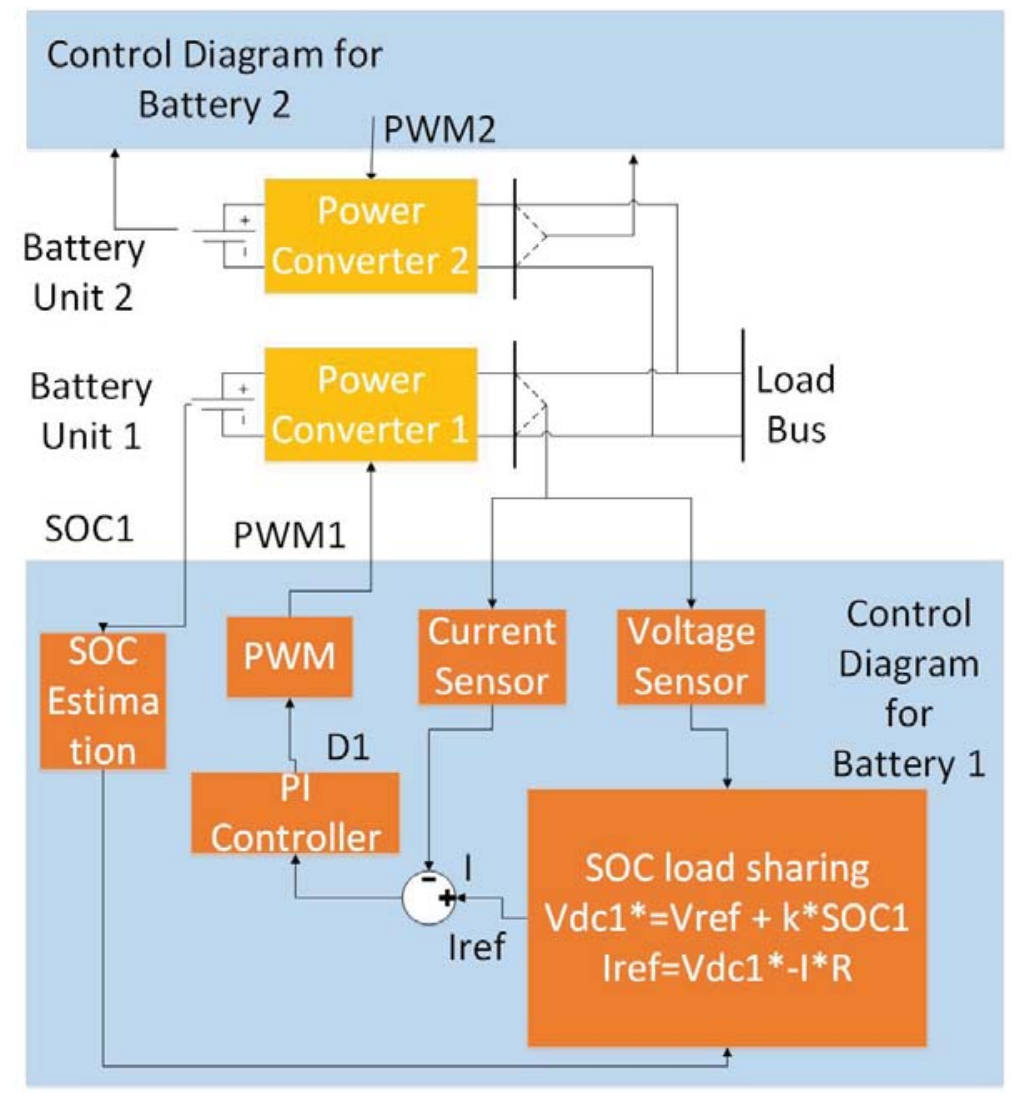

Figure 8: SoC control method diagram in blocks

The SoC control method presented in this thesis is similar to the one mentioned in [17], that they all tend to balance the SoC by modifying the droop equation based on their SoC state. This method requires that the converter controller has a SoC estimator for its battery, so that they could adjust their droop curve based on that. The reference voltage regulation equation of this method 
for each converter is

$$
V_{r i}=V_{\text {set }}+\alpha_{i} S o C_{i}
$$

where $V_{r i}$ is the current open circuit reference voltage, and $\alpha_{i}$ stands for the $i_{t h}$ SoC modification factor.

In this method $\alpha_{i}$ is acting as a 'droop' factor for SoC in this scheme, its value affects SoC balancing speed of the system. According to this equation, the battery with a higher SoC would have a higher reference voltage which would in turn leads to delivering more power to the system. Several limitations should be taken into consideration to choose a proper factor. These rules and corresponding effects will be discussed in Section4.5. Diagram of the system is shown in Fig.9.

Because the bus voltage is related to the battery SoCs, so there is no complete steady state for this system. But during the PI adjusting process (seconds), the battery SoC would only change little $(<0.1 \%)$, therefore the SoCs could be treated as constant during that short period of time. The steady state of this system is defined under such assumption. Despite the transient and switching process of the system, the steady state equations of the system are

$$
\begin{aligned}
V_{r 1} & =V_{\text {set }}+\alpha_{1} S o C_{1} \\
V_{r 2} & =V_{\text {set }}+\alpha_{2} S o C_{2} \\
i_{1} & =\frac{V_{r 1}-V_{\text {bus }}}{R_{1}} \\
i_{2} & =\frac{V_{r 2}-V_{\text {bus }}}{R_{2}} \\
i_{1}+i_{2} & =\frac{V_{\text {bus }}}{R_{\text {load }}},
\end{aligned}
$$




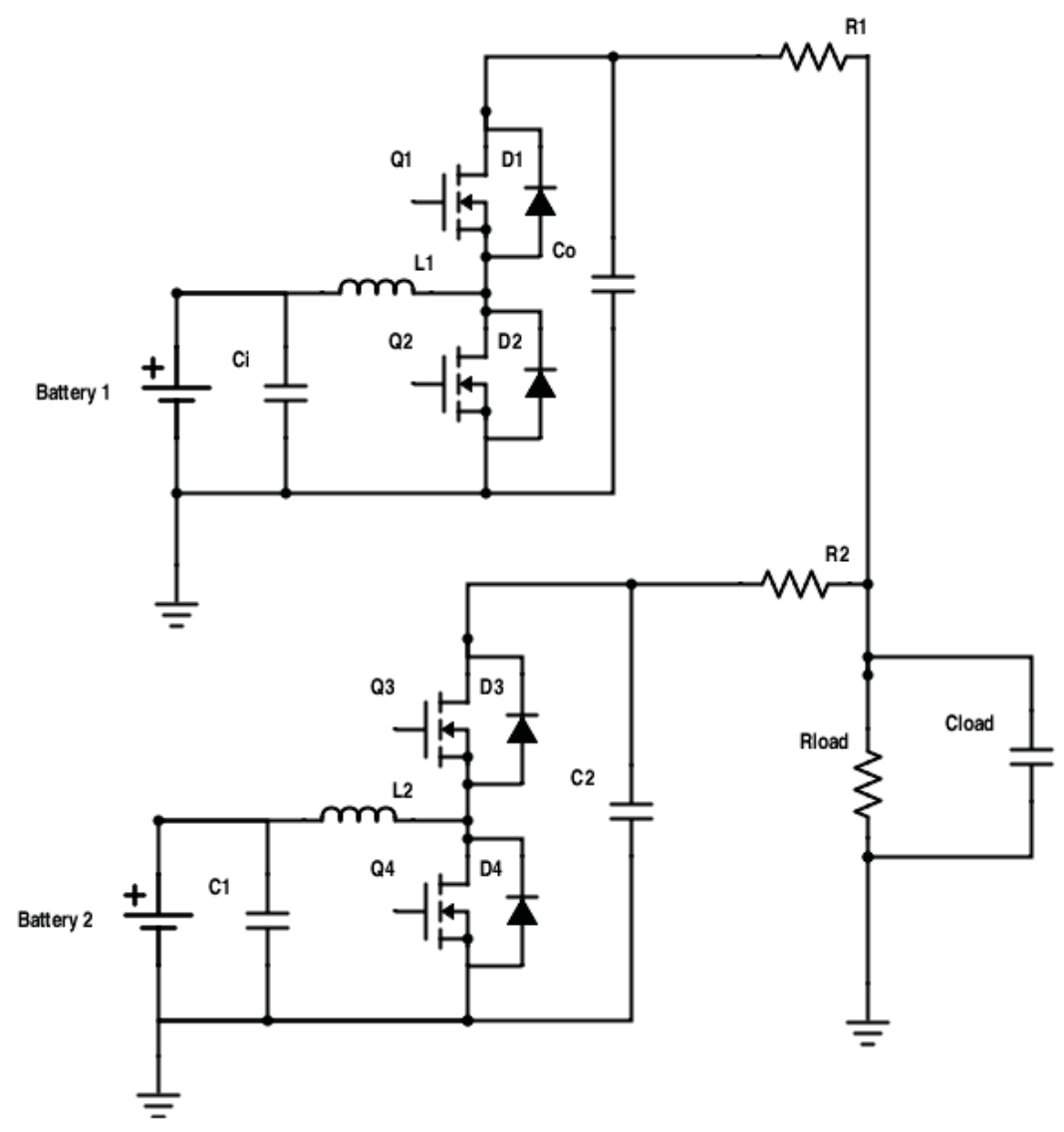

Figure 9: Two power sources with one constant impedance load

where $i_{1}$, and $i_{2}$ are converter output currents, $R_{\text {load }}$ is the load resistant, $V_{\text {bus }}$ is system bus voltage.

Assuming the voltage drop on bus wire can be ignored, two converters have the same SoC factors and virtual series resistant $R_{1}=R_{2}=R$, the output power of each converter and their difference could be given from former equations as 


$$
\begin{aligned}
& P_{1}=\frac{(\alpha S o C 1+V s)(\alpha(R+\text { Rload }) \text { SoC } 1-\alpha \text { Rload SoC } 2+R V s)}{R \text { Rload }+R(R+\text { Rload })} \\
& P_{2}=\frac{(\alpha S o C 2+V s)(-\alpha \text { Rload } S o C 1+\alpha(R+\text { Rload }) S o C 2+R V s)}{R \text { Rload }+R(R+R l o a d)} \\
& d P=\frac{\alpha(R+\text { Rload })(\text { SoC } 1-S o C 2)(\alpha(\text { SoC } 1+S o C 2)+2 V s)}{R(R+2 \text { Rload })} \\
& d I \quad=i_{1}-i_{2}=D_{1} i_{\text {battery } 1}-D_{2} i_{\text {battery } 2} \\
& =\frac{\alpha(S o C 1-S o C 2)}{R} \\
& V_{\text {bus }}=\frac{2 R_{\text {load }}\left(V_{s}+\alpha(S o C 1+S o C 2)\right.}{R+2 R_{\text {load }}}=\frac{2 R_{\text {load }}\left(V_{s}+\alpha \overline{S o C}\right)}{R+2 R_{\text {load }}},
\end{aligned}
$$

where $P_{1}$ and $P_{2}$ stand for converter output power, $d P$ is the power difference of two batteries, $D_{1}$ and $D_{2}$ are duty cycles of two converters, and $\overline{S o C}$ stands for the average SoC value.

It is seen from (13) and (14) that difference in $\mathrm{SoC}$ value will result in a difference in power output shown in (15). Based on (4), assuming the batteries have identical discharging coefficients, their output power will in turn result in a corresponding SoC dropping, so that the higher SoC battery would have a higher SoC drooping rate. Equation (17) revealed that, as long as the load is stable, the bus voltage is proportional to the average SoC in the system. If the load changes rapidly, the bus voltage can not reflect the SoC precisely. But if the load resistant $R_{\text {load }}$ in the system is much larger than the virtual resistant $R$, meaning $R_{\text {load }}>>R$, then the equation (17) is

$$
V_{\text {bus }}=V_{s}+\alpha \overline{S o C}
$$

So the voltage is proportional to the average $\mathrm{SoC}$ if the load resistant is much larger than the virtual resistant. 
A PI controller was applied in this model to regulate the converter output current, with the duty cycle control equation

$$
\begin{array}{r}
D_{i}=a \int\left(\frac{\left(V_{s}+\alpha S o C_{i}\right)-V_{\text {batttery } i}}{R_{i}}-i_{\text {measured }}\right) d t+\ldots \\
\ldots+b\left(\frac{\left(V_{s}+\alpha S o C_{i}\right)-V_{\text {batttery }} i}{R_{i}}-i_{\text {measured }}\right)+D_{0}
\end{array}
$$

where $D_{0}$ is the initial duty cycle, $i_{\text {measured }}$ is the measured current value. Assuming the battery voltage is stable, and the SoC dropping speed of each battery is much slower than the PI regulating space(meaning SoC does not change much during the time PI controller regulates current to its reference), the average model for this system could be written as

$$
\begin{aligned}
L_{1} \frac{d i_{L 1}}{d t} & =V_{1}-D_{1} u C_{1} \\
C_{1} \frac{d u C_{1}}{d t} & =D_{1} i_{L 1}-\frac{u C_{1}-V_{\text {bus }}}{R_{1}} \\
L_{2} \frac{d i_{L 2}}{d t} & =V_{2}-D_{2} u C_{2} \\
C_{2} \frac{d u C_{2}}{d t} & =D_{2} i_{L 2}-\frac{u C_{2}-V_{\text {bus }}}{R_{2}} \\
D_{1} i_{L 1}+D_{2} i_{L 2} & =\frac{V_{\text {bus }}}{R_{\text {load }}},
\end{aligned}
$$

where $C_{1}$ and $C_{2}$ are output capacitors of converters, $L_{1}$ and $L_{2}$ are inductors in bidirectional converters.

The parameter setting for this analysis are :Substitute in the parameters applied in this model showed in Table I; set bus voltage reference to $110 \mathrm{~V}$; set initial SoC to 0.8 for battery 1 and 0.7 for battery 2; PI controllers regulate the 
output current as shown in $i_{\text {out }}=\frac{V_{\text {ref }}+\alpha S o C-V_{\text {bus }}}{R}$; and the battery voltages are set to $50 \mathrm{~V}$. Next, the solution is found from

$$
\begin{aligned}
D_{1} i_{L 1} & =\left(\text { Vref }+\alpha S o C 1-V_{\text {bus }}\right) / R 1 \\
D_{2} i_{L 2} & =\left(\text { Vref }+\alpha S o C 2-V_{\text {bus }}\right) / R 2 \\
0 & =D_{1} i_{L 1}-\frac{\left(50 / D_{1}-V_{\text {bus }}\right)}{R 1} \\
0 & =D_{2} i_{L 2}-\frac{\left(50 / D_{2}-V_{\text {bus }}\right)}{R 2} \\
V_{\text {bus }} & =\left(D_{1} i_{L 1}+D_{2} i_{L 2}\right) R_{\text {load }},
\end{aligned}
$$

and the values of variables at the equilibrium point are given as: $D_{1}=0.438596, D_{2}=$ $0.440529, i_{L 1}=6.89561 A, i_{L 2}=5.73037 A, V_{\text {bus }}=110.976 \mathrm{~V}$.

The state matrix of this system is

$$
A=\left(\begin{array}{cccc}
0 & -\frac{D_{1}}{L 1} & 0 & 0 \\
\frac{\frac{R_{\text {load }}}{R 1}+D_{1}}{C_{1}} & -\frac{1}{C_{1} R_{1}} & \frac{D_{2} R_{\text {load }}}{C_{1} R_{1}} & 0 \\
0 & 0 & 0 & -\frac{D_{2}}{L_{2}} \\
\frac{D_{1} R_{\text {load }}}{C_{2} R_{2}} & 0 & \frac{\frac{R_{\text {load }} D_{2}}{R_{2}}+D_{2}}{C_{2}} & -\frac{1}{C_{2} R_{2}}
\end{array}\right)
$$

Substitute the parameter values, the eigenvalues of this matrix could be calculated. That the eigenvalues of this matrix are

$$
-9980.64,-19.3587,-5000-7244.29 i,-5000+7244.29 i \text {. }
$$

The system is stable if all its eigenvalues' real parts are negative [19]. From the result above that all of the eigenvalues are located in the left half of the plane, thus the system is stable at this equilibrium operating point.

The linear stability analysis including the battery charging and discharging process was not done. Because, assuming the battery is supporting a load, 
Table 1: System element Parameter I

\begin{tabular}{|c|c|c|c|}
\hline Item & Symbol & Value & Unit \\
\hline Capacitor & $C_{1}$ & 1000 & $\mu F$ \\
\hline Capacitor & $C_{2}$ & 1000 & $\mu F$ \\
\hline Inductor & $L_{1}$ & 1 & $m H$ \\
\hline Inductor & $L_{2}$ & 1 & $m H$ \\
\hline Droop factor 1 & $R_{1}$ & 0.1 & $\Omega$ \\
\hline Droop factor 2 & $R_{2}$ & 0.1 & $\Omega$ \\
\hline Load Resistor & $R_{\text {load }}$ & 20 & $\Omega$ \\
\hline
\end{tabular}

there is no steady equilibrium point for battery SoC, as the system goes it will discharge the battery until it hit its capacity limitation.

\subsection{SoC balancing speed}

By solving (8)-(12) and substituting the solution of battery current to (4) the difference of SoCs changing speed could be given as $v 1=\frac{d S o C 1}{d t}$ and $v 2=\frac{d S o C 2}{d t}$

$$
\begin{aligned}
v 1-v 2 & =\frac{d S o C_{1}}{d t}-\frac{d S o C_{2}}{d t}=-\frac{i_{\text {battery } 1}}{C_{e 1}}-\frac{i_{\text {battery } 2}}{C_{e 2}} \\
& =-\frac{\alpha\left(R+R_{\text {load }}\right)(S o C 1-S o C 2)\left(\alpha(S o C 1+S o C 2)+2 V_{s}\right)}{C_{e} R\left(R+2 R_{\text {load }}\right) V_{i}} .
\end{aligned}
$$

From this equation it can be seen that the difference of SoCs, SoC1 - SoC2, determines the difference of v1 and v2. The battery with a higher SoC would have a higher SoC decreasing speed. And when batteries' SoCs become identical their SoC decreasing speed will be the same, though their SoC drooping speed depend on the coefficient, $C_{e}$, load resistant, $R_{\text {load }}$, and bus voltage, $V_{i}$, as well. The factor $\alpha$ could affect the SoC tracking speed too; the larger factor, the faster balancing speed this system could have.

The above analysis is done based on the assumption that two batteries were 
identical, in real application such assumption may not be true. The discharging efficiency would vary based on battery type, discharging voltage and surrounding temperature [20]. If the charging coefficient of the batteries are different, (30) would become

$$
\begin{aligned}
& \frac{d S o C_{1}}{d t}-\frac{d S o C_{2}}{d t}= \\
& \frac{1}{R\left(R+2 R_{\text {load }}\right) V_{i}}\left(\frac{\left(\alpha S o C_{1}-V_{s}\right)\left(\alpha(R+R l o a d) S o C_{1}-\alpha R_{\text {load }} S o C_{2}+R V_{s}\right)}{C_{e 1}} \ldots\right. \\
& \left.\ldots-\frac{\left(\alpha S o C_{2}+V_{s}\right)\left(-\alpha R_{\text {load }} S o C_{1}+\alpha\left(R+R_{\text {load }}\right) S o C_{2}+R V_{s}\right)}{C_{e 2}}\right) .
\end{aligned}
$$

Then substitute $S o C_{1}=S o C_{2}$ into (31) and solve for $C_{e 1}$ results in

$$
C_{e 1}=-\frac{C_{e 2}\left(\alpha R S o C_{2}+R V_{s}\right)}{-\alpha R S o C_{2}-R V_{s}}=C_{e 2} .
$$

It shows that at the point where battery SoCs are identical, to have the same SoC, the battery discharging coefficients have to be the same. Difference in them would cause bias for SoCs balancing under this condition. This phenomenon would be observed and discussed in the simulation section.

\subsection{Limitation on SoC droop Factor}

Although a large SoC coefficient $\alpha$ could increase the SoC balancing speed, there are several limitations in a practical system on its choosing, which includes bus voltage regulations, battery current limits, and transmission power limits.

1. Bus voltage regulation requirement.

Most loads require a stable voltage on bus line. According to different requirements of load and system stability, the permitted operating range of volt- 
age would vary $[21,22,23]$. Assuming the required voltage regulation range is $\pm u \%$ and the virtual resistant is $R$, with a battery current limitation $I_{i n-\max }$ and $I_{\text {out-max }}$ the factor of SoC should fulfill

$$
\begin{gathered}
\alpha+I_{\text {charge-max }} R<u V_{s} \\
I_{\text {discharge-max }} R<u V_{s} .
\end{gathered}
$$

\section{Battery output current limitation.}

Due to capacity and material condition limitation, battery has a limitation for its maximum output power, with regular working status it is marked as current limitation [24]. Assuming the battery rated voltage is $V_{\text {battery }}$ and has a current limitation as $i_{\max }$, consider the worst situation that one fully charged battery is delivering power to the load, the factor of SoC should fulfill

$$
\frac{V_{s}+\alpha}{R_{\text {load }}+R}<i_{\max } .
$$

Simplifying the inequality (35) to

$$
\alpha<\left(\left(R_{\text {load }}+R\right) i_{\text {max }}-V_{s}\right) .
$$

this limitation ensures safety when a fully charged battery is plugged into a system with minimum operating voltage.

\section{Transmission power limitation.}

According to (15), with a high enough SoC control factor $\alpha$, in order to balance the $\mathrm{SoC}$ one of the batteries will try to deliver power to another, meaning $i_{1} i_{2}<0$. One of the reasons to avoid this result is the poor energy transfer efficiency from one energy storage to another, during which both charging 
and discharging process contain considerable power loss. It is also highly recommended to minimize this kind of operation [25] because it would shrink the battery's lifetime [26]. By solving (8)-(12) the formula of $i_{1}, i_{2}$ could be given. Assuming battery 1 has a larger initial SoC and its difference with battery 2 is $d S o C$, to make sure $i_{2}>0$ the SoC factor must fulfill

$$
\alpha<\frac{R V_{s}}{d S o C R_{\text {load }}-R S o C_{2}} .
$$

Above are the three major limitations on SoC factor choosing. All of them were considering the worst operating conditions, from the simulation it can be seen that aside from these extreme conditions, the SoC factor has a wider choosing range.

\subsection{Bus voltage-SoC detection}

One advantage this control scheme possessed could be seen from (18), that the bus voltage changes linearly along with the system average SoC value. For load and power source sides, the operator could easily get the information of the system SoC average status without communication lines using equation

$$
\overline{S o C}=\frac{V_{b u s}-V_{s}}{\alpha}
$$

Information needed are the bus voltage value, SoC droop factor $\alpha$ and initial reference voltage $V_{s}$, which could be given to the operator when setting up the system. To get an accurate SoC average value there are several requirements:

1. Load resistant has to be stable: if the load oscillates too severely or heavily, (17) can not be simplified to the equation above.

2. The operator have access to the value of each batteries' SoC droop factor 
$\alpha$ and initial reference voltage $V_{s}$.

3. Batteries share similar charging/discharging coefficient: because a large difference in charging coefficient would result in balance bias(discussed in section 4.4), the SoC estimation would have some bias (this effect could be mitigated by adjusting droop factor, introduced in section 5.2.2).

Generally, as long as the system is well designed and functioning well, this method could be applied and work smoothly. Application making use of this feature would be demonstrated in section 5.5.

\subsection{Method summary}

In this section the proposed SoC balance droop control method was presented. The models used during the study were discussed and demonstrated, which including battery, bi-directional DC boost/buck converter and controller system. Mathematical analysis on feasibility and stability on this method were done, the results showed the studied system applying this method is stable at the desired operating point. Under proper settings this method could effectively balance the SoC of all batteries in a parallel connected power system, additionally information of the average SoC of all batteries in system could be put into the power line in form of bus voltage value. The SoC balancing speed and SoC droop factor limitation were discussed as well, conclusions were that the value of SoC droop factor $\alpha$ has a positive relation with the SoC balancing speed. Due to consideration of practical limitation both on battery and power system, the choice of SoC droop factor is limited. Results also showed that the difference in batteries discharging coefficients could affect the SoC balancing, this feature will be discussed again in the following chapter. 


\section{Simulation Study}

The simulation is done using MATLAB/Simulink R2014a, models used in the simulation were built according to the average differential equations as shown in (20)-(24). In this section several different scenarios will be simulated: two batteries support one load; two batteries and one solar cell support one load; two batteries charged by solar cell; impact of different battery charging coefficient on SoC balance; droop factor in mitigating battery coefficient difference issue. The SoC balance results, output current and power of batteries and bus voltage simulated values will be shown and discussed in each scenario. The results show that this method could balance the batteries SoCs well when batteries share similar charging/discharging coefficient, while the fact that bus voltage drops along with average SoC was observed. At last a comparison between conventional droop control and SoC balance control in an insufficient power supply system is demonstrated, showing that the system with SoC balance control could avoid possible bus voltage crush and do reasonable load planing and shedding.

\subsection{Simulation of two batteries support one load}

In this part two identical batteries are connected to the system with different initial SoCs. The simulated diagram is shown in Fig.10. The parameter setting in this simulation is listed in Table II. The values set for inductors guaranteed that the converters were working in continuous mode, the battery charging/discharging coefficients were picked coordinated with the load value so that the battery would not run out of energy too fast, yet still has an considerable drop in SoC.

The output power and SoC of batteries are shown in Fig.11 and Fig.12. From 


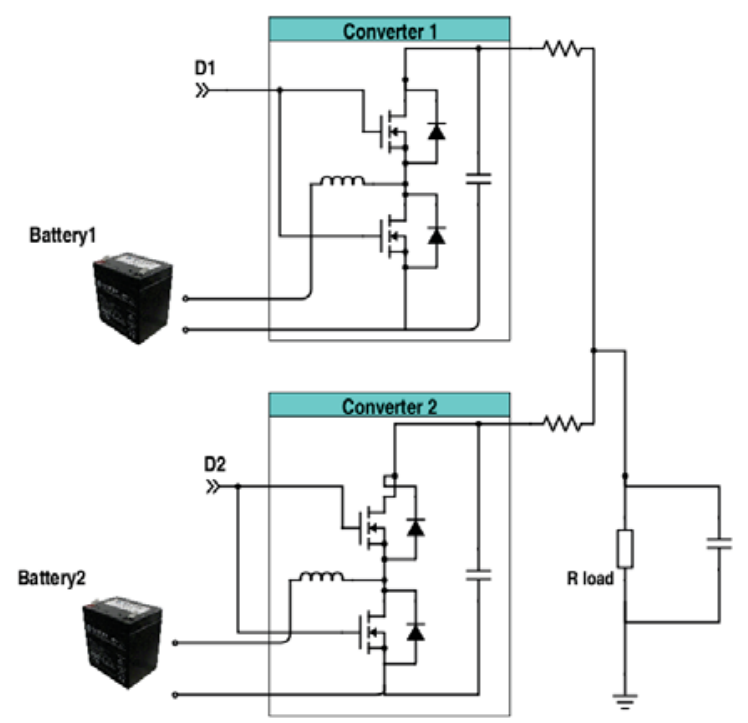

Figure 10: Simulation system diagram

these figures it can seen that the two SoC values were well balanced. There was some bias in the end caused by bus series resistor but is relatively small. At the very beginning there was some negative current goes into battery 1 , this is due to a large SoC factor $\alpha=10$, which was chosen to make a faster balancing speed, if the factor was chosen to make sure there will be no input current for batteries the factor should be no larger than 2 (calculated using (37)) but the tracking speed would be much slower.

Table 2: Simulation Parameter II

\begin{tabular}{|c|c|c|c|}
\hline Item & Symbol & Value & Unit \\
\hline SoC1 & $S o C_{1}$ & 40 & $\%$ \\
\hline SoC2 & $S o C_{2}$ & 50 & $\%$ \\
\hline Battery 1 capacity & $C_{e 1}$ & 10000 & $\mathrm{~A} \mathrm{~s}$ \\
\hline Battery 2 capacity & $C_{e 2}$ & 10000 & $\mathrm{~A} \mathrm{~s}$ \\
\hline Bus Series Resistor & $R_{s}$ & 0.001 & $\Omega$ \\
\hline SoC factor & $\alpha$ & 10 & $\mathrm{~N} / \mathrm{A}$ \\
\hline Load Resistor & $R_{\text {load }}$ & 20 & $\Omega$ \\
\hline
\end{tabular}




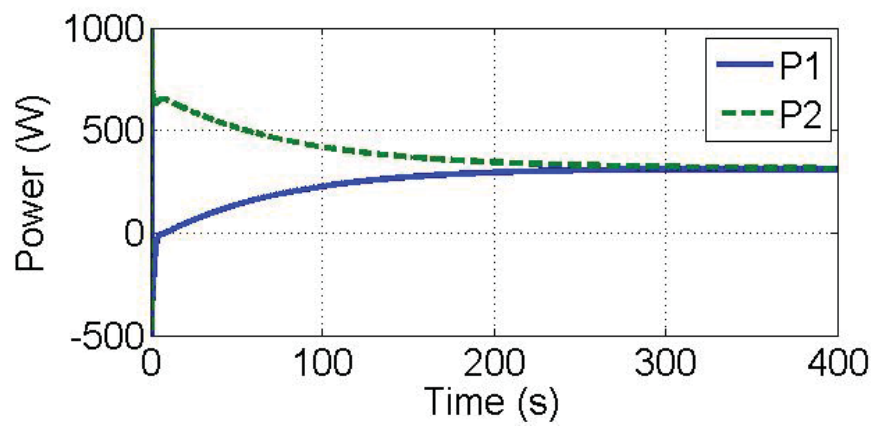

Figure 11: Power output of batteries

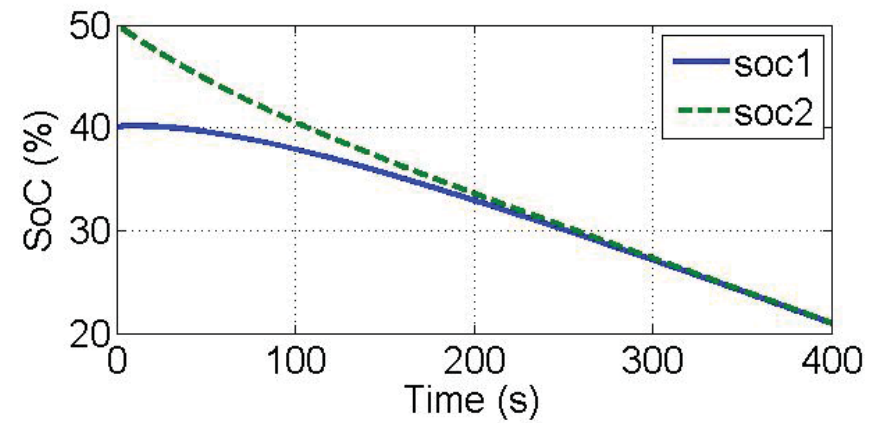

Figure 12: SoC of batteries 


\subsection{Simulation of batteries with different charging coefficient}

\subsubsection{Impact of difference in battery charging coefficient}

As mentioned in section 4.4, with different charging/discharging coefficient two batteries can not reach identical SoC status. By solving equation

$$
\frac{i_{\text {battery } 1}}{C_{e 1}}=\frac{i_{\text {battery } 2}}{C_{e 2}}
$$

the difference of SoC at the point where SoC differential are same for both batteries can be given. The solution of (39) has a complicated form as

$$
\begin{gathered}
\Delta S o C=\frac{\alpha C_{e 1} R_{\text {load }} S o C_{2}-\alpha C_{e 2} R_{\text {load }} S o C_{2}+2 C_{e 2} R_{2} V_{s}+C_{e 1} R_{\text {load }} V_{s}+C_{e 2} R_{\text {load }} V_{s}}{2 \alpha C_{e 2}\left(R_{2}+R_{\text {load }}\right)} \ldots \\
-\frac{\sqrt{4 C_{e 1} C_{e 2} R_{1} R_{2}+4 C_{e 1} C_{e 2}\left(R_{1}+R_{2}\right) R_{\text {load }}+\left(C_{e 1}+C_{e 2}\right)^{2} R_{\text {load }}^{2}}\left(\alpha S o C_{2}+V_{s}\right)}{2 \alpha C_{e 2}\left(R_{2}+R_{\text {load }}\right)}-S o C_{2} .
\end{gathered}
$$

The charging coefficient of battery 1 is 10000 and battery 2 is 5000. Assuming they reached balanced $\mathrm{SoC}$ when $\mathrm{SoC}_{2}=0.3$ and substituting the data in table II, it can be given that $S o C_{1}$ would be 0.318 under such setting. There is a 0.018 bias of SoCs between batteries under this setting. One could use (40) to estimate the SoC difference at the steady point reached applying this control method.

The simulation results applying different battery discharging coefficients are shown in Fig. 13 and Fig. 14, from these graphs it can be seen that the estimated gap between batteries SoC values when they reached an identical SoC decreasing status. The green dot line represents the higher discharging factor battery, showing it tried to output less power in order to stay at a similar SoC level with another battery, which can be seen in Fig. 14. 


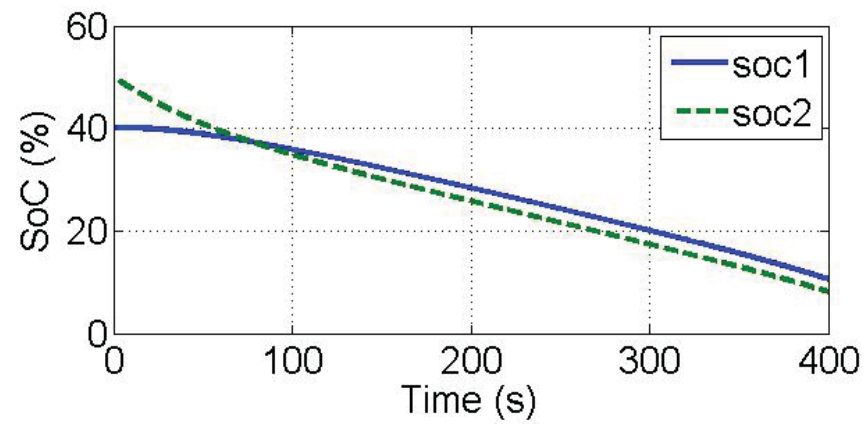

Figure 13: SoC of batteries with different Ce

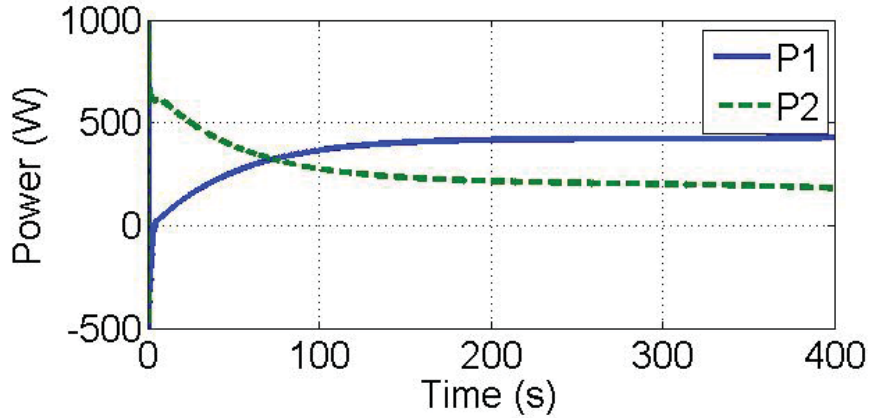

Figure 14: Output power of batteries with different $\mathrm{Ce}$ 


\subsubsection{Droop factor compensation for battery discharging coefficient difference}

As (30) indicated, there is no way to re-balance the SoC by modifying SoC droop factor $\alpha$ when two batteries don't share a same discharging coefficient, but another tunable factor, the outer current droop factor, $R$, could affect the output power as well. As mentioned in background section, converters applying droop control regulate their output current based on load condition and droop factor too, a smaller droop factor shapes a flatter droop curve, and leads to more power output under same voltage level, this feature could be used to compensate for unbalance SoC states caused by different discharging coefficients. Take (39) and solve for $R_{1}$ assuming $S o C 1-S o C 2=0$, the result is

$$
R_{1}=\frac{C_{e 2} R 2}{C_{e 1}}
$$

The system simulated is the same batteries-load system as shown in Fig.10. Substitute the parameter used in previous section, it can be given that the over all droop factor for converter 1 should be half the value of converter 2 , meaning $R_{1}=\frac{1}{2} R_{2}$. To validate this compensation effect, the previous simulation was done again applying the new droop factor (In the model the total output factor consists of the virtual factor and the real wire resistant; in the calculation they were summed up). The SoC portraits and the converter output power are shown in Fig.15 and Fig.16, from the SoC figure it can be seen that the gap between SoCs has been narrowed, yet the SoCs are still not fully converged. This might be caused by one of the assumption made when solving (41), that the SoC dropping balanced point $\mathrm{SoC}_{2}$ is unknown actually, what applied is the value got from previous simulation result. To fully address this problem further work is needed. 


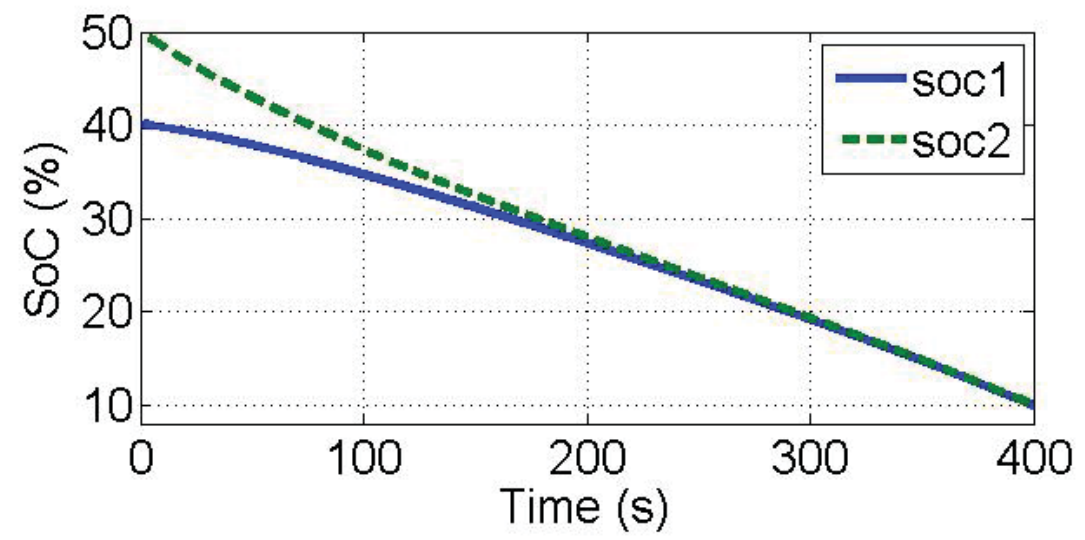

Figure 15: SoC: Different battery coefficient with droop compensation

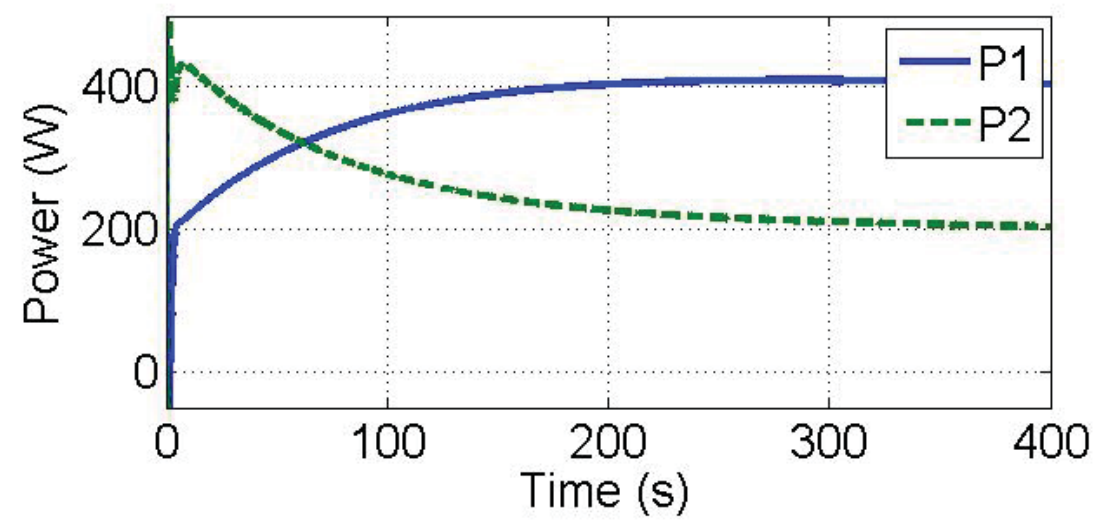

Figure 16: Power: Different battery coefficient with droop compensation 


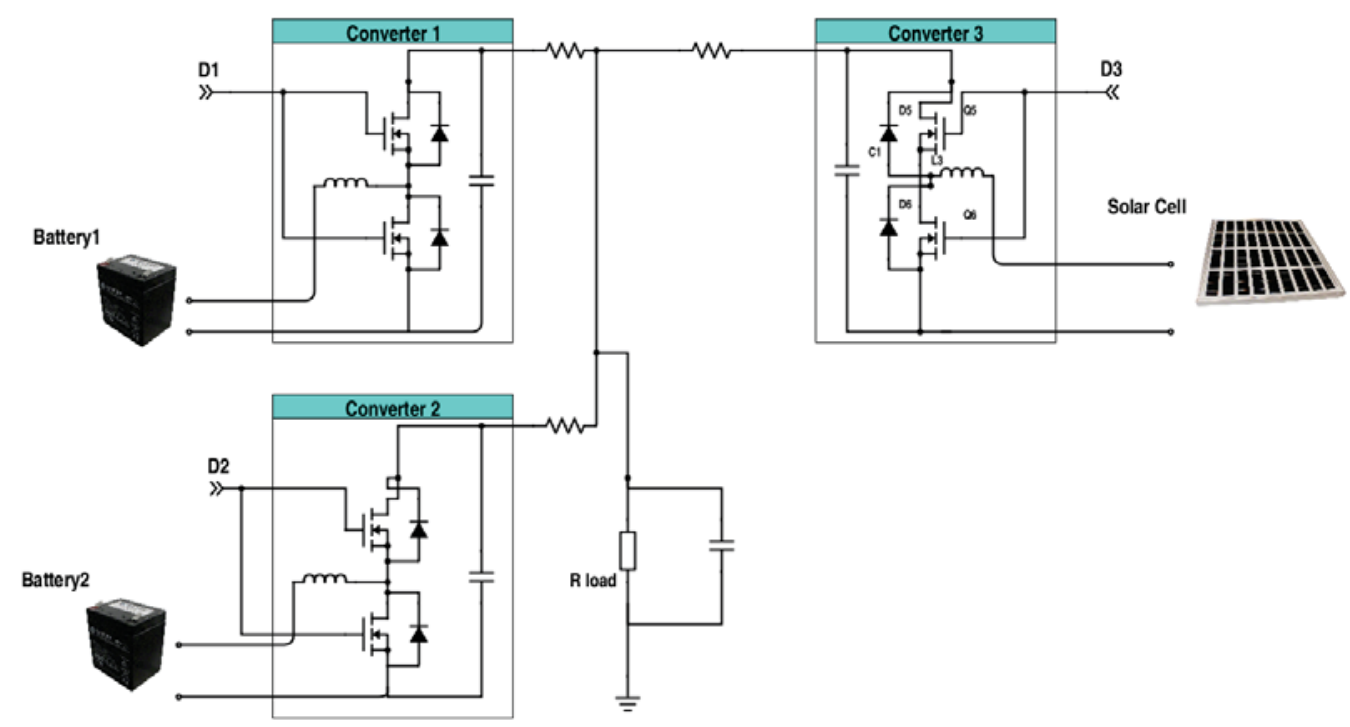

Figure 17: System with batteries and source

\subsection{System with power source}

In previous chapters the system under study had storage and load only, but in a practical system it is most likely to have some other power sources. For example, in a self-sustained community system the possible energy sources could be solar panel and wind turbine generator [27]. To test this control strategy with other power sources, a solar cell with rated power of $380 \mathrm{~W}$ was connected to the system shown in Fig. 17. The operation rule for this solar cell is to deliver constant power to the load when bus voltage is under maximum tolerated value. The simulation results are shown in Fig.18 and Fig.19. Still a large $\alpha$ was chosen to see a faster tracking.

From the figures it can be seen that the two batteries were powering the load based on their own SoC status: battery 1 with a lower initial SoC was delivering less power than battery 2 at the beginning, and after $100 \mathrm{~s}$, when two batteries SoCs are converged, their discharging power became identical.

Another simulation regarding charging process is performed as well, in this 


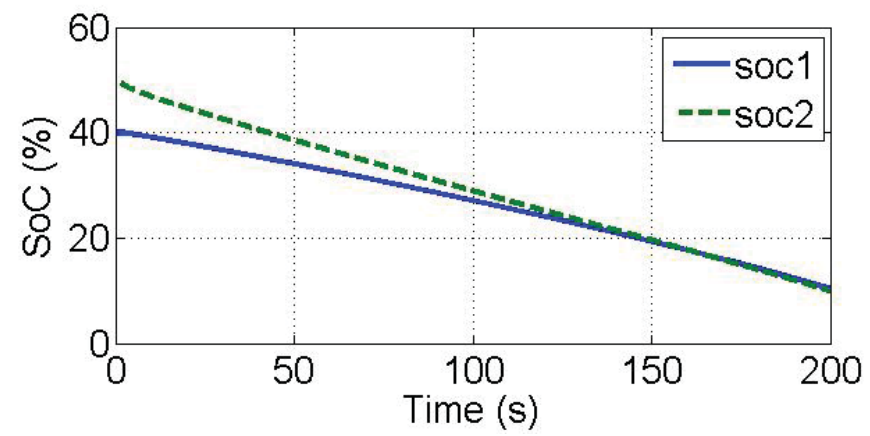

Figure 18: SoC of batteries with sources

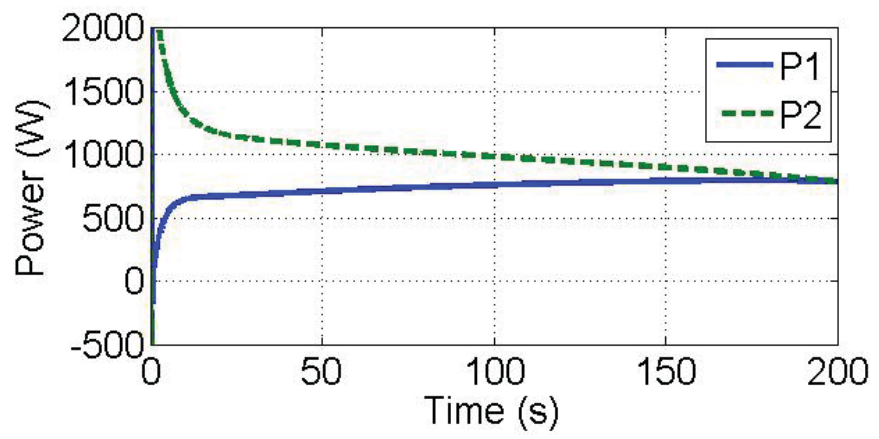

Figure 19: Power of batteries with sources 
case the load requirement was set to be less than the solar cell rated power thus the two batteries should be charged based on their SoC status. The simulation results are shown in Fig.20 and Fig.21. From Fig.20 it can be seen that the two battery SoCs were converged in the end, but before $100 \mathrm{~s}$ the SoC of battery 2 was dropping while battery 1 SoC was increasing meaning that battery 2 was outputting power to the system while battery 1 was being charged. The power difference could be seen in Fig.21 as well, that solar cell was charging both batteries after 100s, and before that the battery 2 is trying to deliver power to reach identical SoC due to the choice of alpha again, eventually both batteries were charged equally.

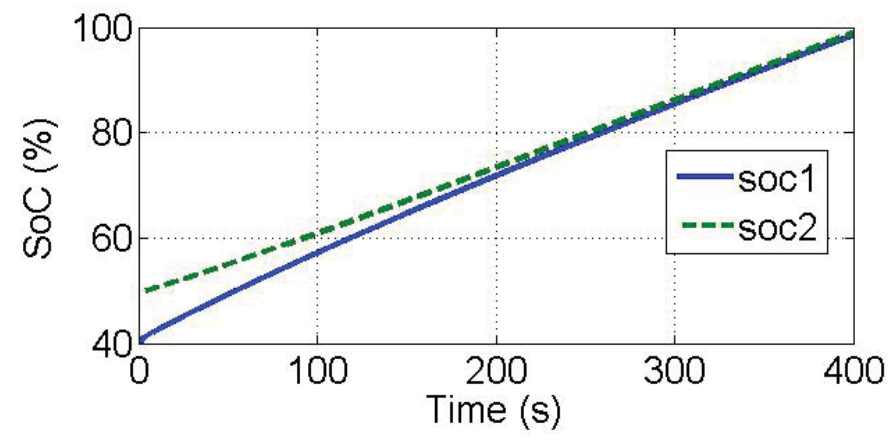

Figure 20: SoC of batteries charging with sources

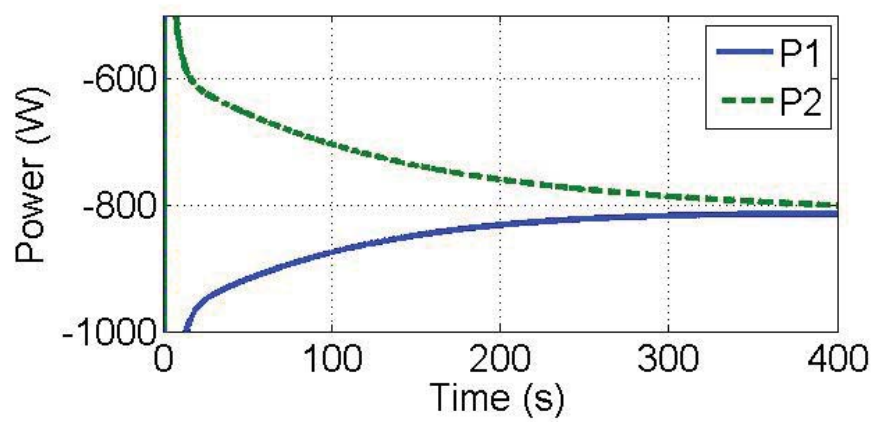

Figure 21: Power of batteries with sources 


\subsection{Simulation on charging/discharging switch process}

In order to observe the transaction using this method when battery switches from charging to discharging status, a simulation concerning this scenario was made. In this simulation the solar cell was delivering sufficient power covering the load at the beginning, but after 200 second it was shut down, and after that batteries have to support the load. The initial battery SoC setting is the same with the former batteries-solar cell-load system. Battery 1 had a lower initial SoC.

The simulation results for the battery SoCs are shown in Fig.22, the power converter output shown in Fig.23 and the bus voltage shown in Fig.24. From the figures it can be seen that the batteries were charged based on their $\mathrm{SoC}$ status at the beginning, battery 1 was receiving more power. After 200s, solar is off and two batteries have to support the load, and they shared the load based on their SoC states and kept on approaching identical SoC status. In the end the SoCs were converged. But from the bus voltage figure Fig.24 it can be seen that there is an obvious voltage step change at $\mathrm{t}=200 \mathrm{~s}$, this was caused by the wire resistant $R_{b}$ us and virtual resistant $R_{i}$. The reference output voltage of converters were not changed during the whole process, but as the current flows direction reversed suddenly along converter output wires, there would be a sudden reverse voltage drop on the virtual resistant between converter output port and the bus. This phenomenon does not disturb the SoC balancing process, but could affect the accuracy of SoC-voltage detection, which will be introduced in the following section. 


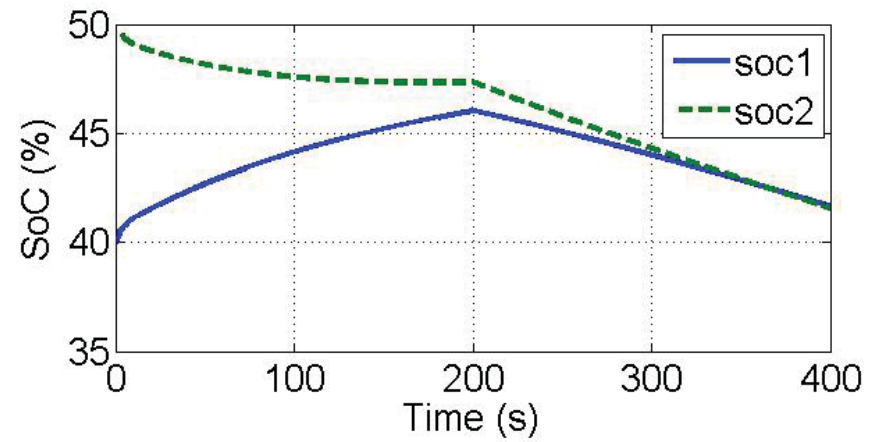

Figure 22: SoC of batteries in charging/discharging switching scenario

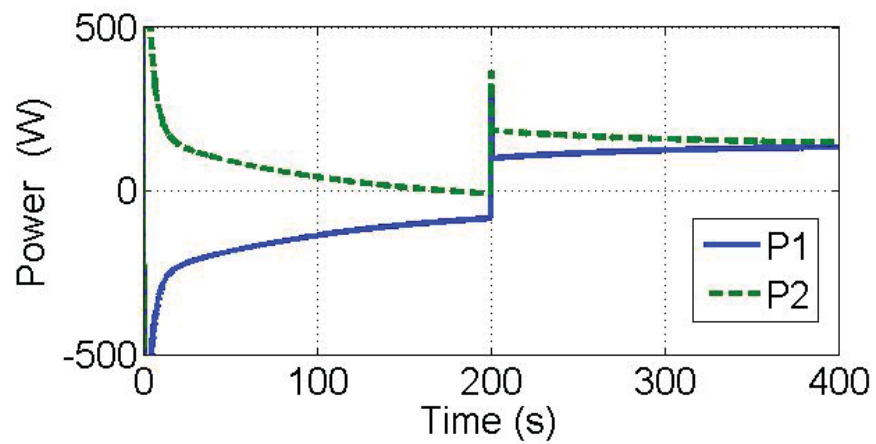

Figure 23: Power of batteries in charging/discharging switching scenario

\subsection{Application of $V_{b u s}-S o C$ detection}

In this section the feature that this method could put $\mathrm{SoC}$ information on the system bus voltage will be demonstrated, to observe this effect a compare between SoC control and droop control was made. The simulated system was the same as shown in Fig.10, the initial SoCs of batteries were set to 0.5 and 0.4. A conventional droop control was applied to the system with a droop coefficient $R=1$, the bus voltage and SoC status are shown in Fig.25 and Fig.26, from the figures it can be told that the bus voltage barely changed throughout the time. Because the droop control equation $V=V_{s}-a I$ indicates that the output voltage would only change along with the load, from both views of the load and 


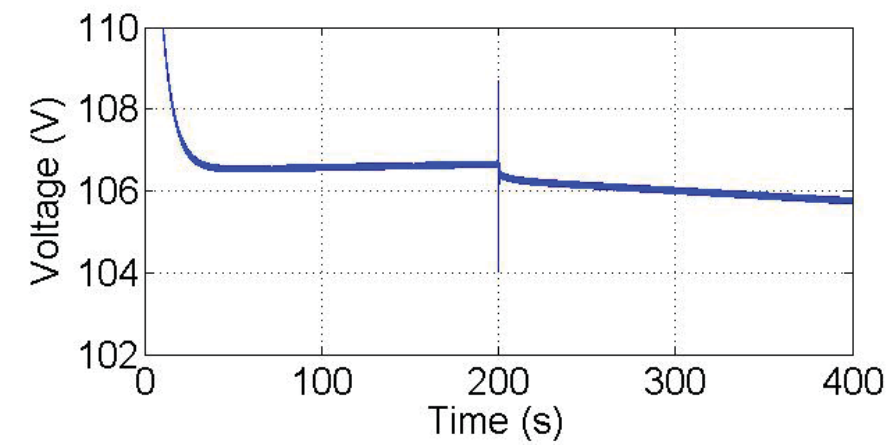

Figure 24: BuS Voltage in charging/discharging switching scenario

source sides there is no information of batteries' SoC status.

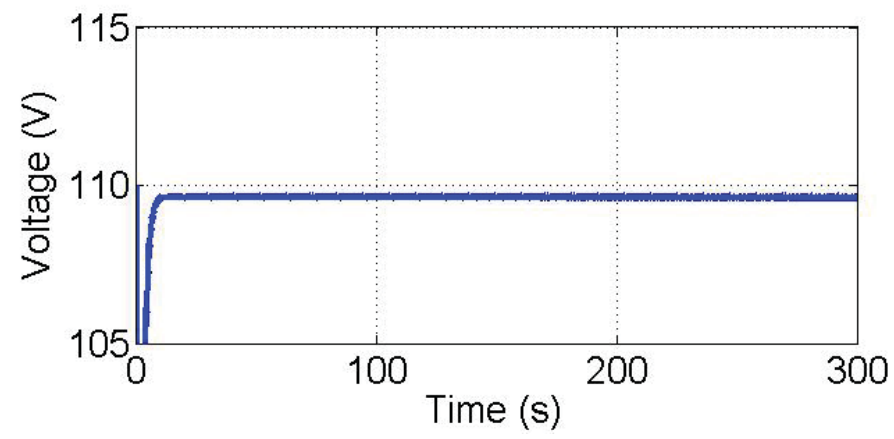

Figure 25: Bus voltage with droop convention control

The simulation of system with SoC control are shown in Fig.27 and Fig.28. As the figures showed, the bus voltage drops along with the system average SoC. Therefore, by measuring the bus voltage, operators of load or source side can get an over all information about the SoC status of the whole system and make decisions accordingly. In the scenario simulated if the system operator wants to maintain the battery SoC at certain level or make sure the system works within voltage regulation range long enough until outer energy source being plugged in, it may be a wise choice to shed loads. One interesting feature is that if the battery was fully charged the bus voltage would be higher than its desired value.

It is vital to keep tracking SoC of batteries in some systems while operating, 


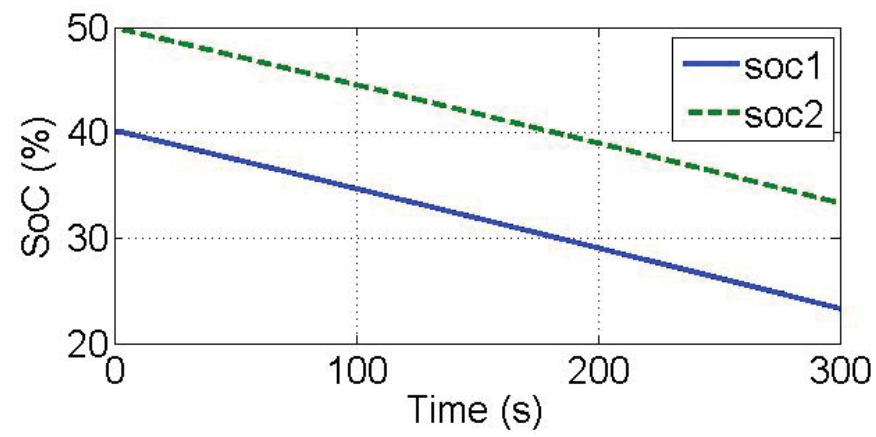

Figure 26: SoC of batteries with convention droop control

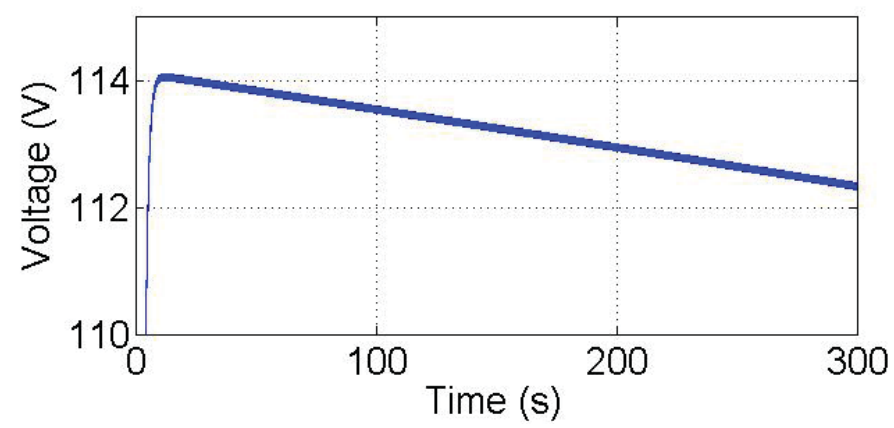

Figure 27: Bus voltage with SoC control

conventional solution would be a communication platform covering the whole system [28], which gathers information form all batteries, distributed power supplies and loads, and make optimal plan according to estimations including weather, load and power price (when connected to main grid) [29][30]. All of such operations need the data of SoC. By applying this control method, the operator can get access to the SoC information of batteries without communication lines, which could save the cost of building one.

The following simulated scenario is a micro system whose only outer source is a solar cell as shown in Fig.17, and the solar irradiation would vary during the day. A simulation concerning insufficient energy supply conditionis presented. In this simulation, the solar cell was shut off until 200 s, presenting insufficient 


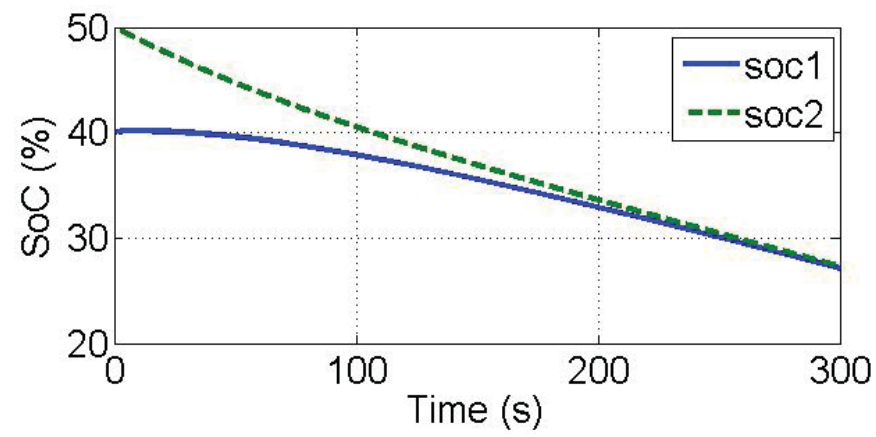

Figure 28: SoC of batteries with SoC control

irradiation times. The two batteries were supposed to support load with a resistant of $25 \Omega$ and survive the insufficient energy time. As shown in Fig. 29, the system experienced a voltage collapse after 130s. And after $200 \mathrm{~s}$, when the solar panel was reconnected to the system with sufficient power supply, the solar panel started to charging both batteries and the bus voltage was raised back to its rated value.

For a load side operator in this situation, there was not much could be done to prevent it. In such condition, the only solution is to cut off the loads. To make a decision on the load shedding, the following information is needed: 1 . Remaining battery stored energy; 2. Load estimation; 3. Time remaining until outer sources reconnected. What the system operator lacks here is the first information, unless a communication system links batteries and load, no load shedding actions could been seen as reasonable. An oversize load shedding could harm the benefit of users and operator, while a insufficient shedding could just lead to another crush as well.

A system using SoC control would have bus voltage value shown in Fig.30. It looks similar at the beginning to the former one, but this time the voltage represented the average battery $\mathrm{SoC}$ status, and at $t=40 \mathrm{~s}$ by doing a simple 
induction based on(38), the load operator could be aware of that all batteries have a SoC below 10\%. With a quick check on load demand and power supply estimation, it is clear to cut certain loads to make sure system survive the insufficient power time. Assuming the lower SoC limitation of battery is $8 \%$, the maximum load current the batteries could support would be $\frac{\Delta S o C}{T C_{e}}=1.25 A$, so the load should be cut to $R_{\text {load }}=88 \Omega$. After 200 s the bus voltage was raising, meaning the solar source was delivering power into the system, thus the shaded load could be reconnected to system again. The process is shown in Fig. 30. Here it can be seen that the system survived the insufficient power period.

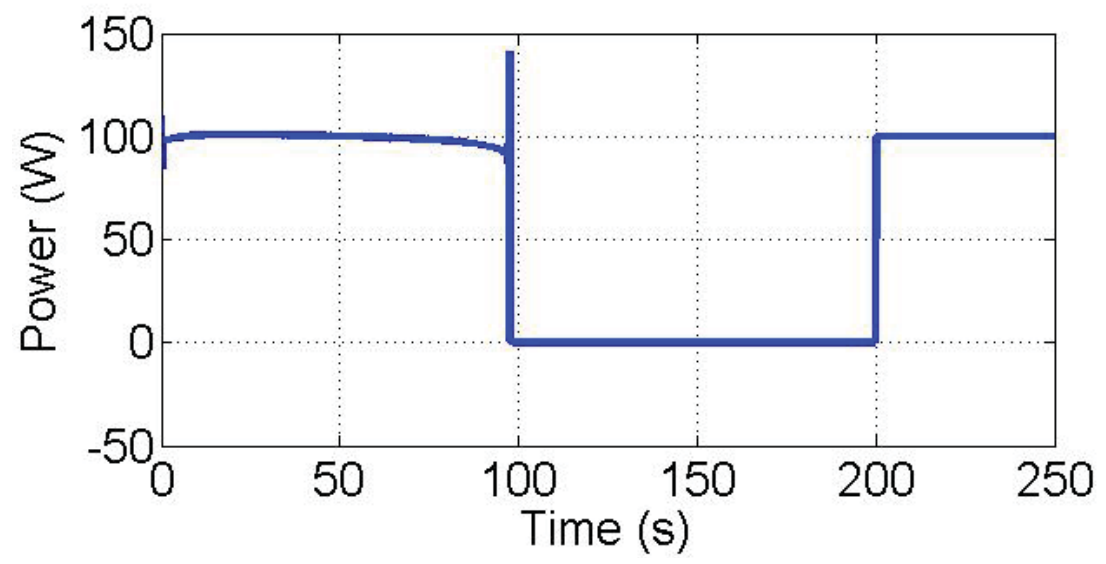

Figure 29: Bus voltage with convention droop control, insufficient power condition, the batteries ran out of all capacity after 130 s and the system was down 


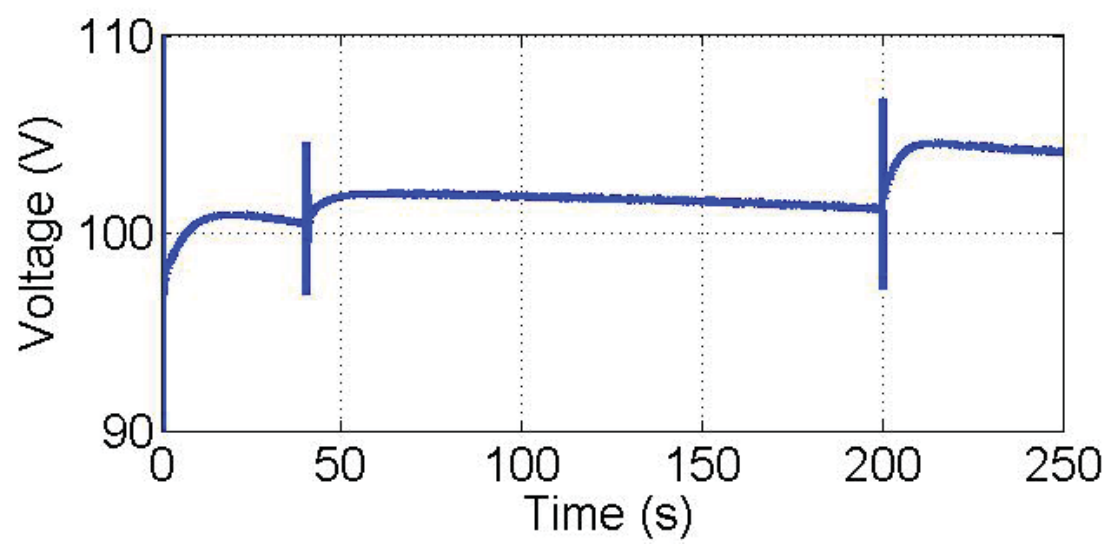

Figure 30: Bus voltage with SoC control, insufficient power condition, at $\mathrm{t}=40 \mathrm{~s}$ the operator noticed that bus voltage was too low, revealing that there was no sufficient capacity left for all loads, after reviewing load plan and solar power estimation some loads had to be cut

\subsection{Simulation summary}

As the above simulations showed, the SoC balance control could effectively share the load based on battery's SoC state, effect of this method is that all batteries connected to bus would share an identical SoC. There is a condition that affect the balancing effect, which is the batteri coefficient: SoC balancing process would have some bias due to the battery charging/discharging coefficient difference. According to (40), a larger $d C_{e}$ would lead to a larger balance bias. Simulation concerning the batteries charging process was done, in the simulation batteries were charged based on their SoC states, the results showed the batteries shared an identical SoC states in the end. Lastly a scenario concerning insufficient power supply in micro system was demonstrated, compared to conventional droop control, SoC control could deliver the system SoC state information to the load side through power line, therefore the load manager could arrange its load plan and shedding based on this information. 


\section{Hardware Test}

In this thesis a hardware experiment was conducted to validate the simulation result and test the performance of the SoC balancing droop algorithm on real devices. The devices used in experiments are: a power electronics drive board, DS1104R\&D controller board and CP1104 I/O board. Their lab screen shot is shown in Fig.31. The circuit diagram of the power electronics drive board is shown in Fig.32; each port was connected to the bus via a bi-direction converter controlled using pulsewidth modulation(PWM) method. During the experiment two scenarios of two batteries with one load (as shown in Fig.9) were demonstrated, and port A1 and B1 were chosen to be connected to power sources representing batteries, while port B2 was connected to a DC motor with speed control. The connection diagram is shown in Fig.32.

The power electronics drive board has two independent 3-phase PWM inverters, which were designed to simulate control of two DC machines. Duty cycle of each pair of converters, PWM frequency, and the duty cycle controlling method could be programmed in its Simulink model. After programming, a set of DSP

files were generated and a virtual DSP controller was set in the computer using Dspace. The controller could gather the data collected in the power electronics board and give out the controlling signal (Duty cycle in this situation) via CP1104 I/O board. The real time current, bus voltage and motor rotation speed were sensed using the embedded sensor on the power electronic drive board.

Real batteries were not applied in this experiment. Instead, two power sources with constant voltage were connected to the board as mentioned. According to Fig.6, the battery's voltage drops little within normal operating range(from 0.4 to 0.9), so the battery could be seen as a constant voltage source in that SoC range. 
The two power sources have been set to have constant output voltage despite their SoC status in this test. This setting provided a virtual battery model with a flat V-SoC curve. Because of that, the hardware test didn't reveal the V-SoC relation of batteries in the experiment results. In the system studied, the values converters try to regulate are their output current, so as long as the converters are working in continuous mode and could regulate their output current to reference value, this limitation of the battery model could be neglected. To get the battery SoC values for the controller, the sensor on the power electric driver board will sample the output current of each converter and send them to the programmed DSP controller via CP1104 I/O board. The embedded calculator in the controller then will calculate the $\mathrm{SoC}$ value using (4) and send duty cycle signals(according to its algorithm) out to the PWM generator in the power electronics drive board via I/O board.

\subsection{Scenario I: Two identical batteries support one load}

Scenario I presented two batteries supporting one constant load; virtual battery 2 was set to have a higher initial SoC. The initial SoC for virtual battery 2 was set to be 0.8 while battery 1 is 0.6 . Calculators programmed in DSP1104 will get the sensed current of the two converters and give out their real time SoC states using (4). To apply the presented method, the droop reference voltages for each battery were modified using (7). The motor controller was set to regulate the motor speed to a constant value using the PI control method. In the experiment, a step change in load side was set to test the system stability under large disturbance, the desired rotating speed changed from 400RPM to $1200 \mathrm{RPM}$ at $t=90 \mathrm{~s}$. The default reference voltage, $V_{\text {set }}$, in (4) was set to $48 \mathrm{~V}$ while the $\mathrm{SoC}$ droop factors $\alpha$ were set to 4 , therefore the bus voltage would be $50 \mathrm{~V}$ when the average SoC 


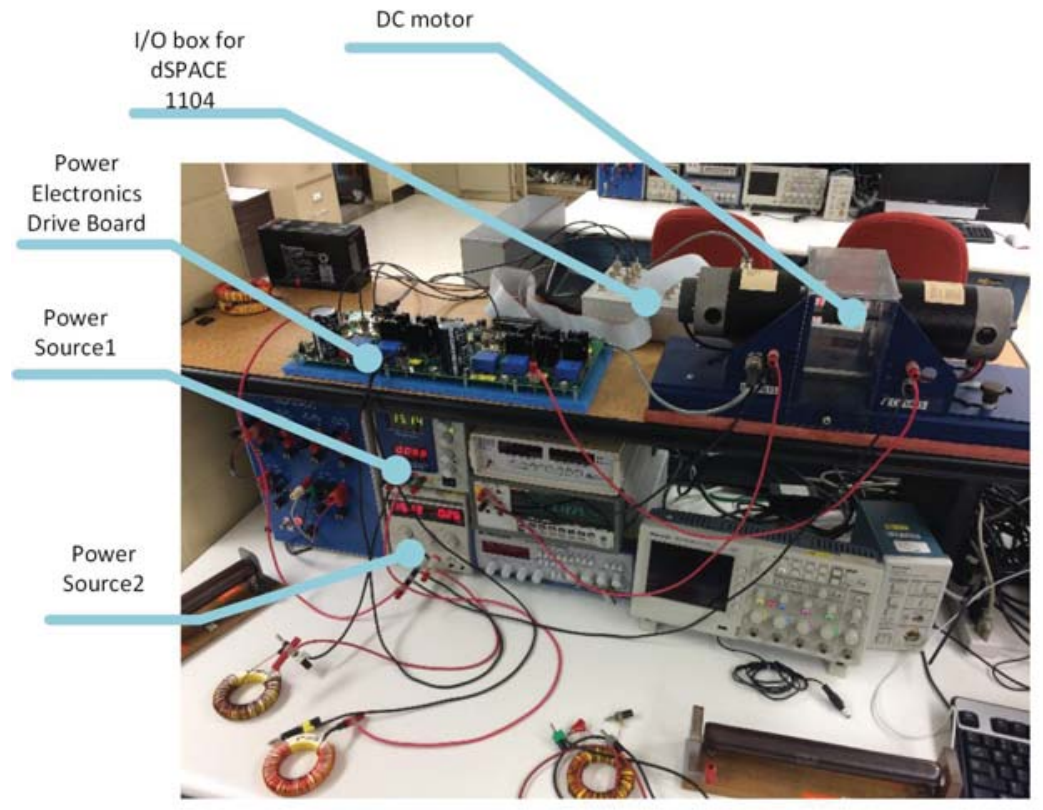

Figure 31: Experiment devices

system is 0.5 . The battery coefficients were set to 200 to have a considerable SoC drop.

The DSP control board has a signal recorder and could transmit its gathered data into a MATLAB data file, the sorted data (including motor rotating speed, bus voltage, battery SoCs, and battery output currents) were exported and shown in Fig.33. To validate the reliability of the data a TDS2014B oscilloscope was connected to capture bus voltage and battery currents; the wave forms of them are shown in Fig.34 and 35.

From Fig.33 it can be seen that the two batteries were trying to reach the same SoC status via regulating their output current. At first, battery 2 had a higher output current (around 0.5A) than battery 1 (near $0 \mathrm{~A}$ ). When two batteries shared the same SoC value, their output current became identical as well. One feature that should be brought up here is that during the time period $0-70 \mathrm{~s}$, battery 1 had some negative current flow, it could be noise or due to a large 


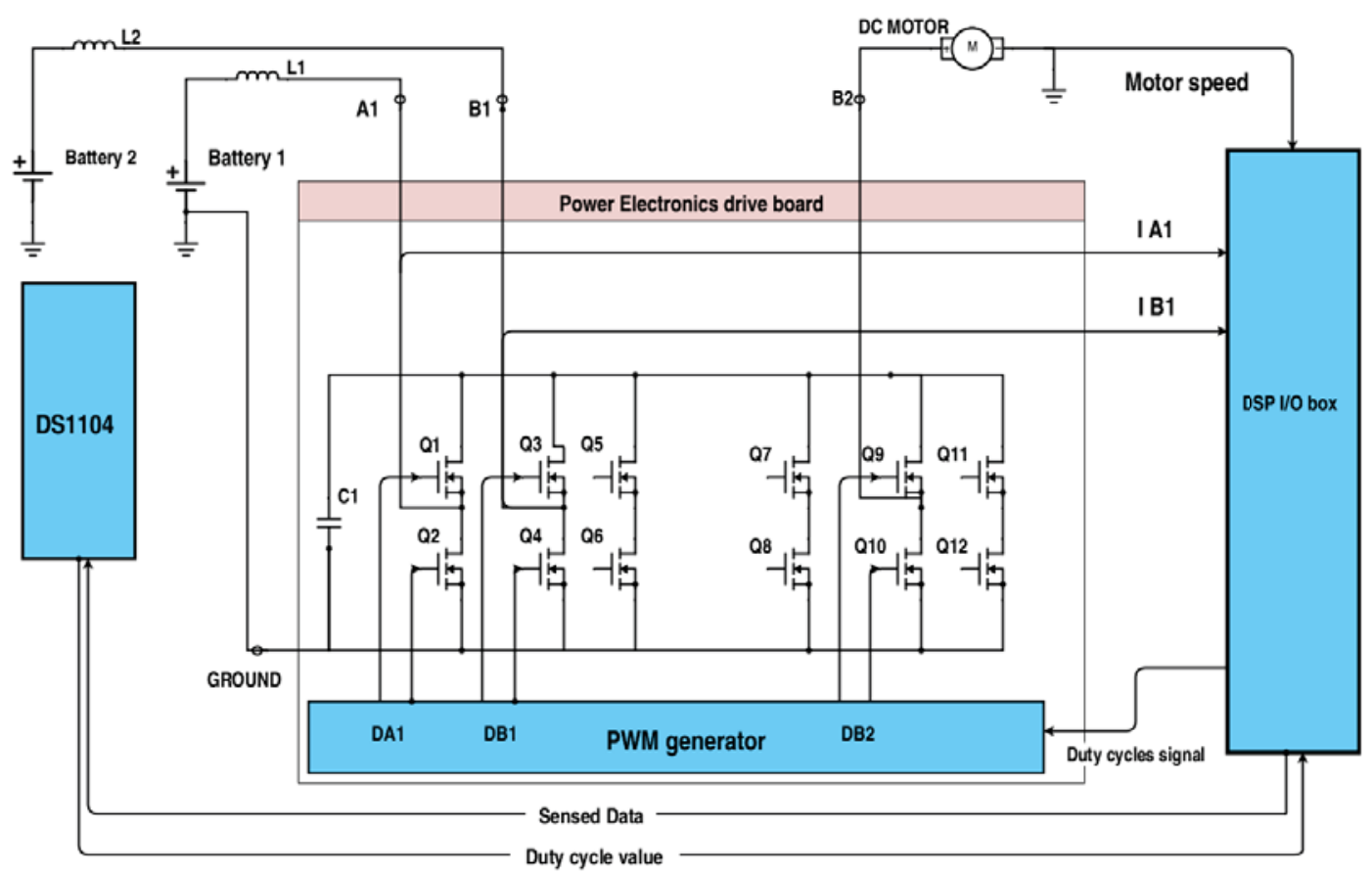

Figure 32: Experiment connection diagram

SoC droop factor $\alpha$. According to the $\mathrm{SoC}$ droop factor limitation discussed in section 4.5, in order not to have transmission power between batteries, a smaller SoC droop factor should be chosen(calculate using (37)).

In Fig.34, the current values gathered from oscilloscope, it can be seen that before $50 \mathrm{~s}$, battery 1 , with a lower SoC, tend not to support the load, whose output current was oscillating around zero. While the other battery, battery 2 , with a higher initial SoC, was driving the motor alone. After $50 \mathrm{~s}$ the motor increased its rotating speed, and it can be seen that there was a step changing of motor speed and current in Fig.33(a),(d) and Fig.34. After around $100 \mathrm{~s}$ the SoCs have been balanced, then the two batteries output currents were converged; the output current shared a similar value. 


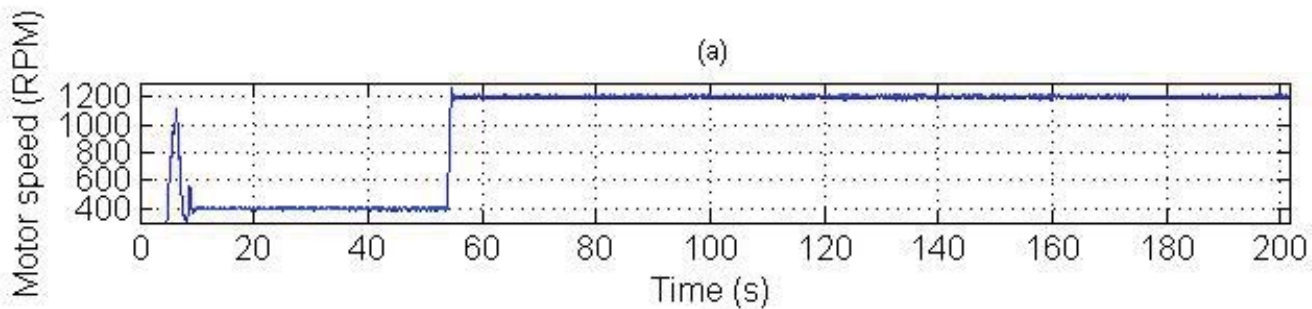

(b)

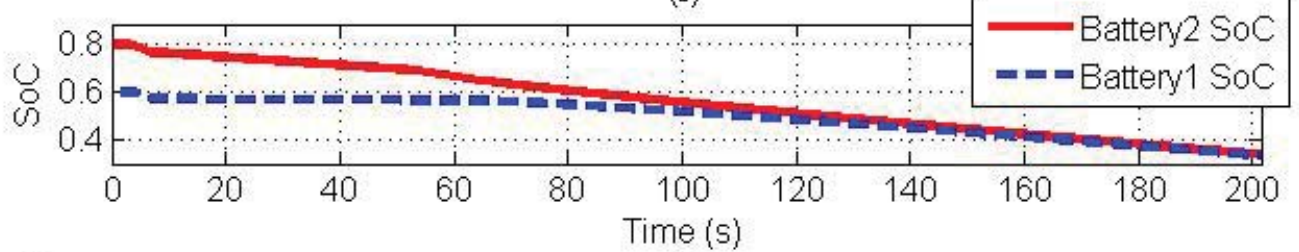

(c)
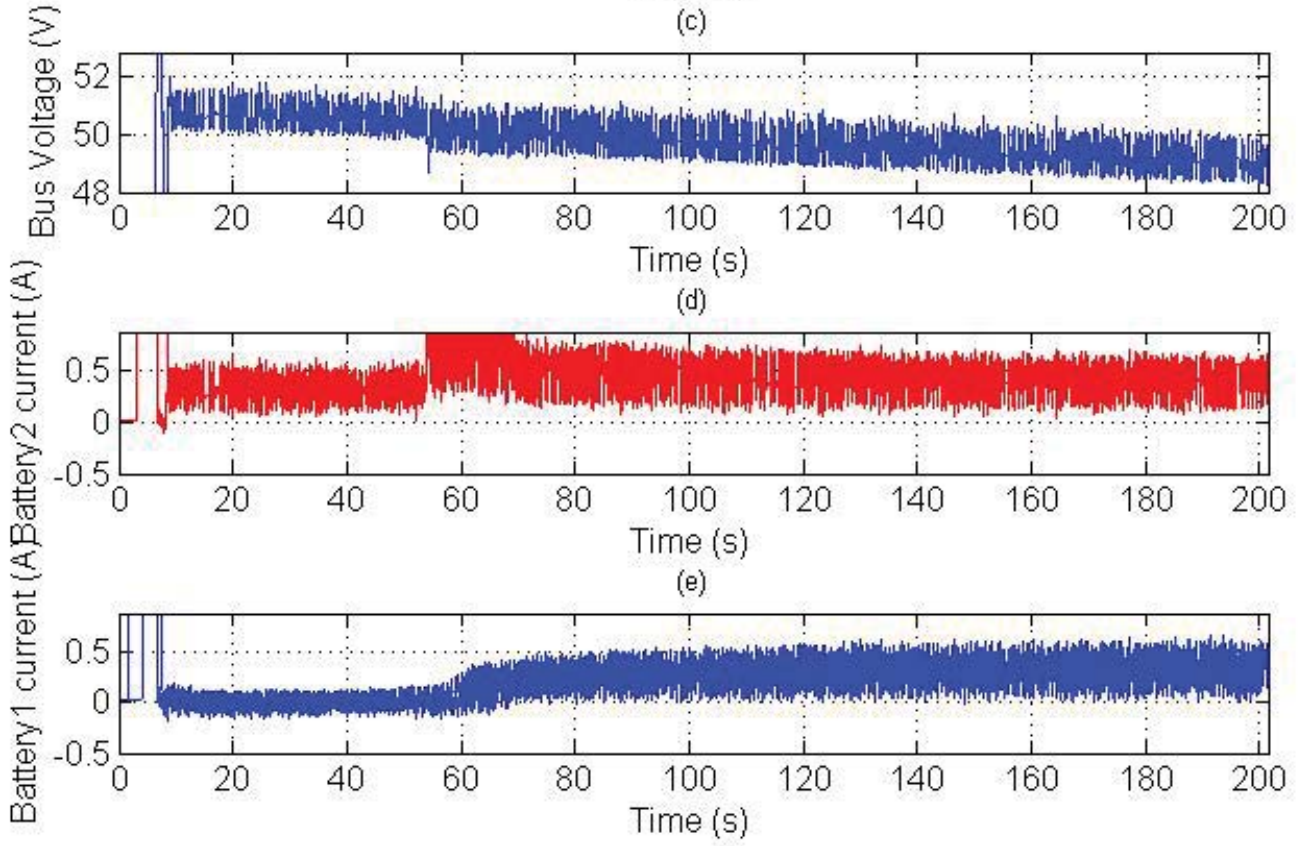

Figure 33: Experiment data, figure(a) showed the motor rotating speed; figure(b) represented SoC values of two batteries, that they were well balanced after 100s; figure(c) is the bus voltage value, drooped along with the SoCs; figure(d) and figure(e) depict the output currents of two batteries, at beginning only battery 2 was delivering power to system, as the load became heavier and SoCs been balanced, battery 1 started increase its output power accordingly 


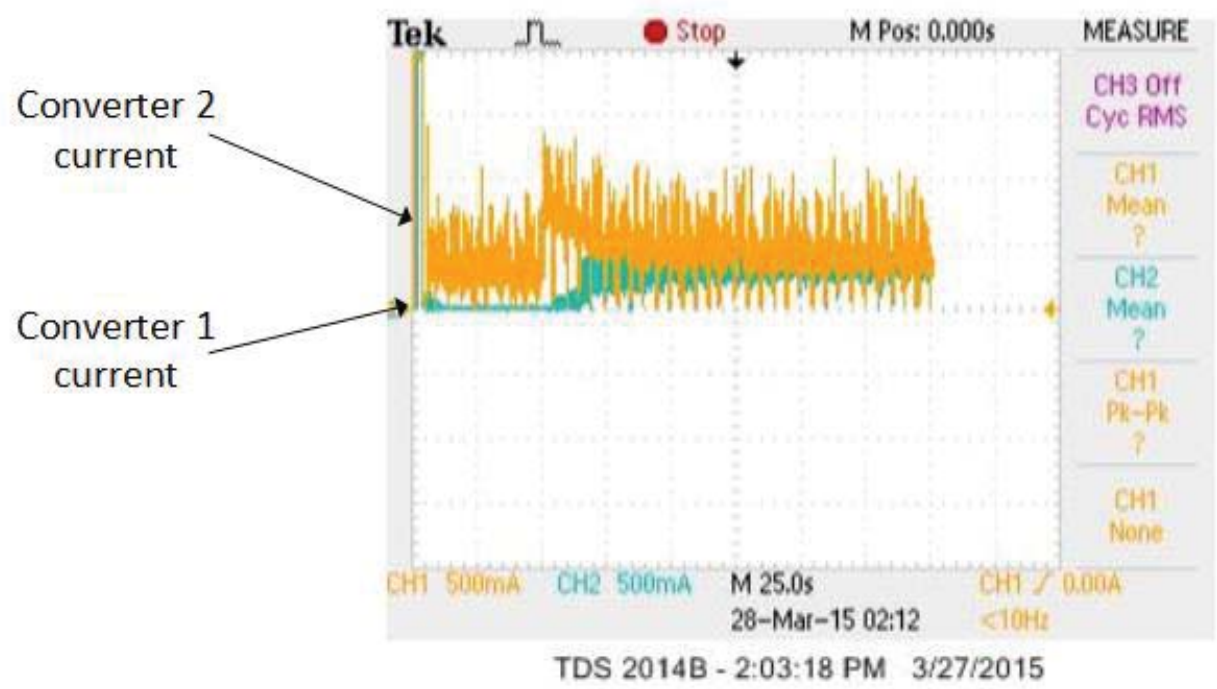

Figure 34: Scenario I: Sampled output current of two batteries in oscilloscope, the yellow line stands for battery 2 current, blue line stands for battery 1 current. Where the yellow arrow pointed is the reference point $(0 \mathrm{~A})$. The output currents were converged after 100s.

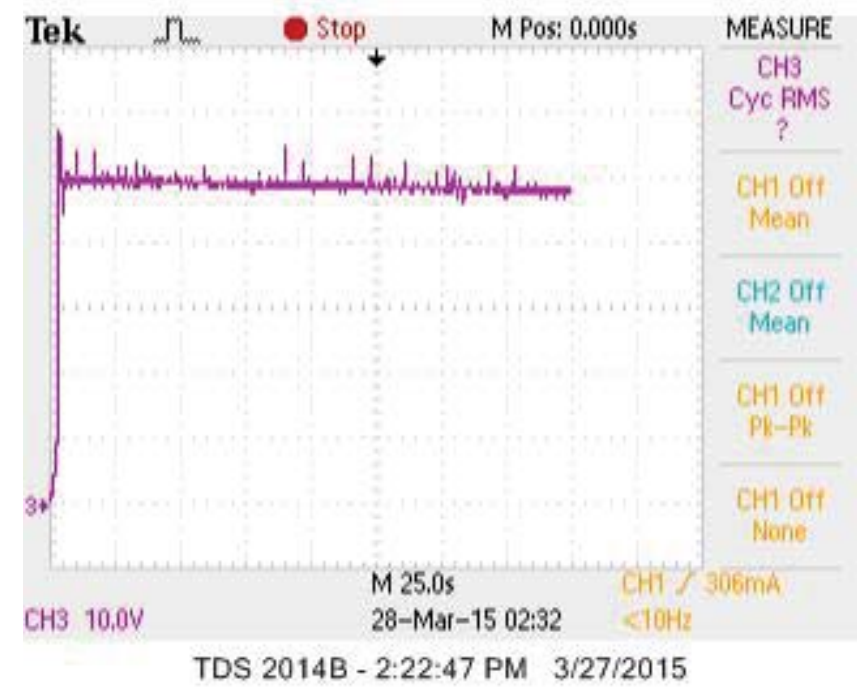

Figure 35: Scenario I: Sampled bus voltage in oscilloscope, voltage was dropping along with the time. 


\subsection{Scenario II: Two batteries support one load with different dis- charging coefficient}

Scenario II studied the impact on SoC balancing cause by difference in battery discharging coefficients. The parameter settings were the same as scenario I, except that battery 1 had a smaller discharging coefficient, $C_{e}=100$, than battery 2. Motor reference speed was set to 1200RPM. According to previous analysis, there will be bias in the $\mathrm{SoC}$ balancing result when two batteries reach the same SoC dropping rate. According to (40) the estimated bias of SoC balance is $\Delta S o C=0.1$; the experiment data gathered by recorder is shown in Fig.36.

From Fig.36(b) it can be seen that there is an obvious gap between the two battery SoCs at the steady states. The blue line represents for current of Battery 1, with a smaller discharging coefficient, laid below battery 2, even though the former battery was delivering less power as could been seen from Fig.36(d)(e). The same phenomenon is observed in the current detected by the oscilloscope in Fig.37, the blue line, representing battery 1 current, was always lower than the yellow line, demonstrating that this battery was trying to give out less power in order to have a slower SoC dropping speed.

A zoomed in view of Fig.36(b) is shown in Fig.39, it can be seen that there is a SoC gap of 0.1 between two SoCs as estimated. To fill the gap one could use the method discussed in section 5.2 .2 by modifying the droop factor.

\section{3 experiment conclusion}

In the hardware test part, two simple battery-load scenarios were tested, the experiment results showed this method could balance the batteries SoCs well under the experimental setting. The condition that batteries with different 


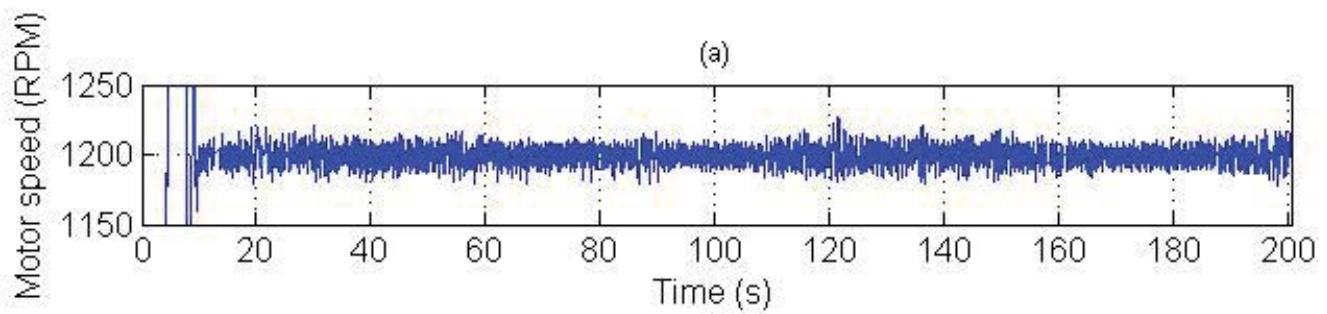

(b)

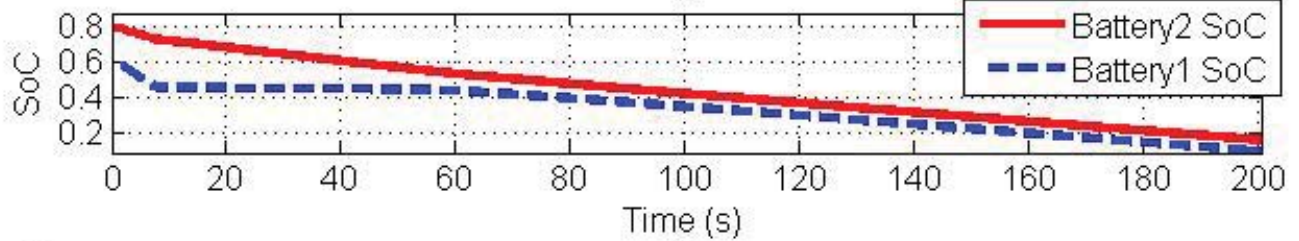

(c)
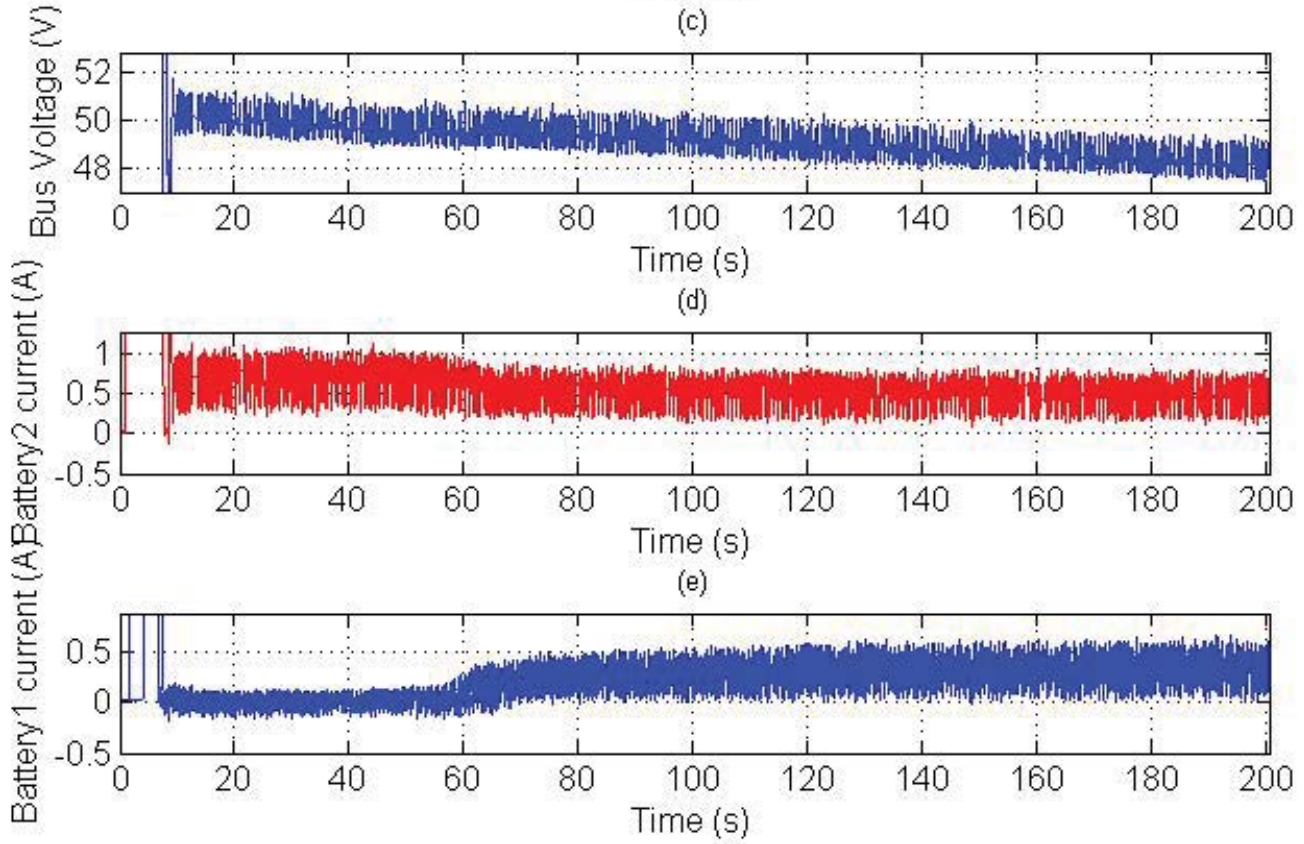

Figure 36: Experiment data of two batteries with different discharging: figure(a) showed the motor rotating speed; figure(b) represented SoC values of two batteries, that there was some bias when the SoC dropping rates were the same after 100s; figure(c) is the bus voltage value, drooped along with the SoCs; figure(d) and figure(e) depict the output currents of two batteries, its difference with scenario $I$ is that after $100 \mathrm{~s}$, battery 1 was still outputting less power 


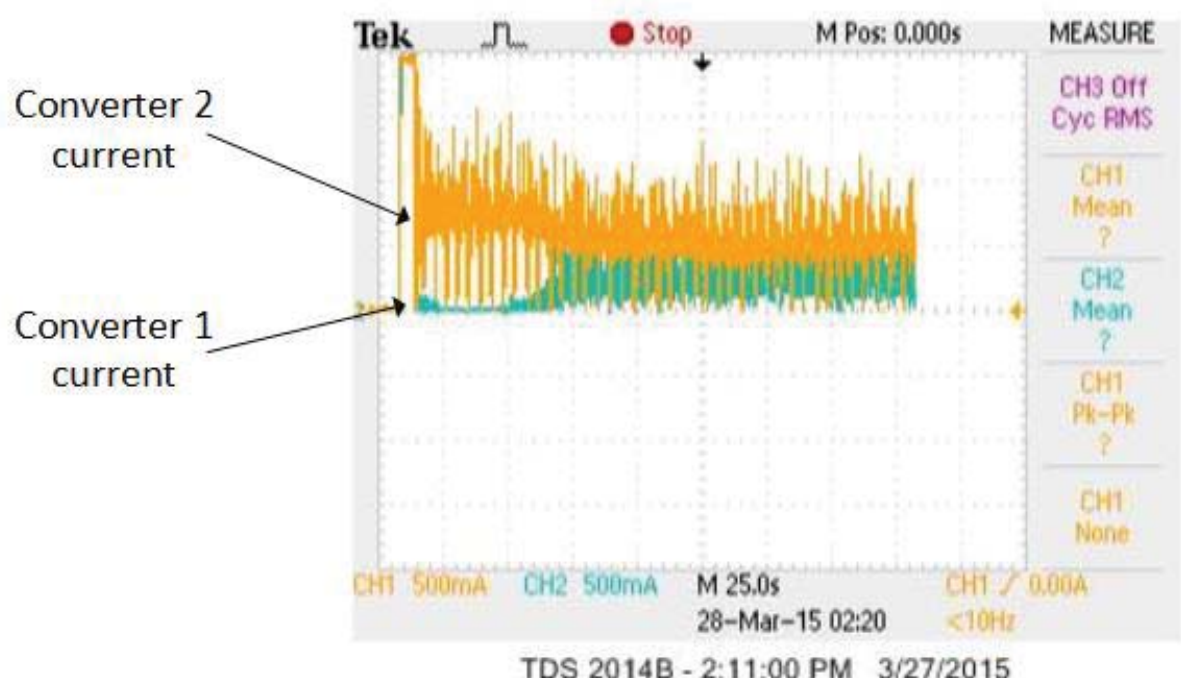

Figure 37: Scenario II: Current data sensed using oscilloscope, green line stands for battery 1 current and yellow line stands for battery 2 . Due to a larger discharging battery 1 was delivering less power in order to save its SoC

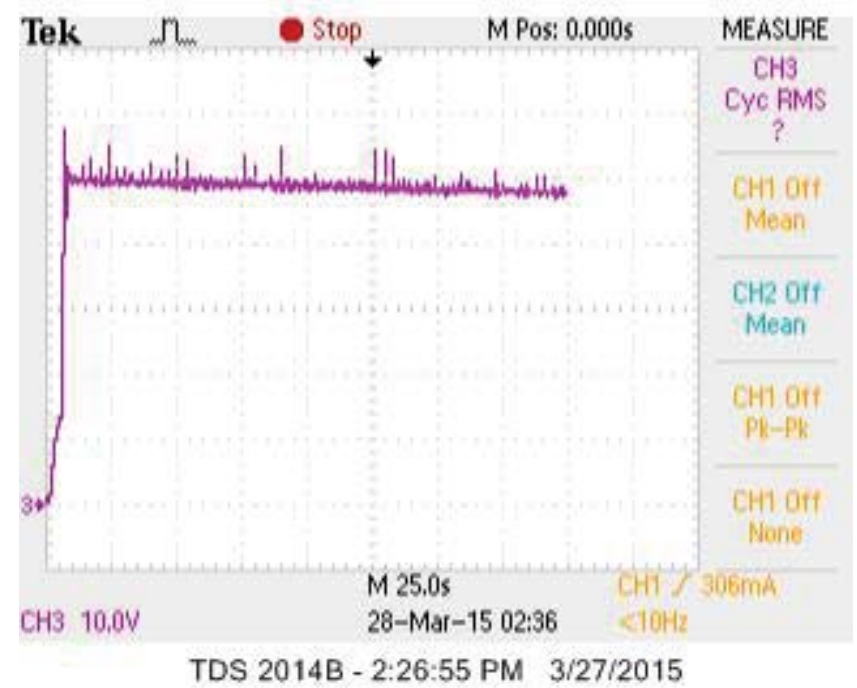

Figure 38: Scenario II: Sampled bus voltage in oscilloscope 


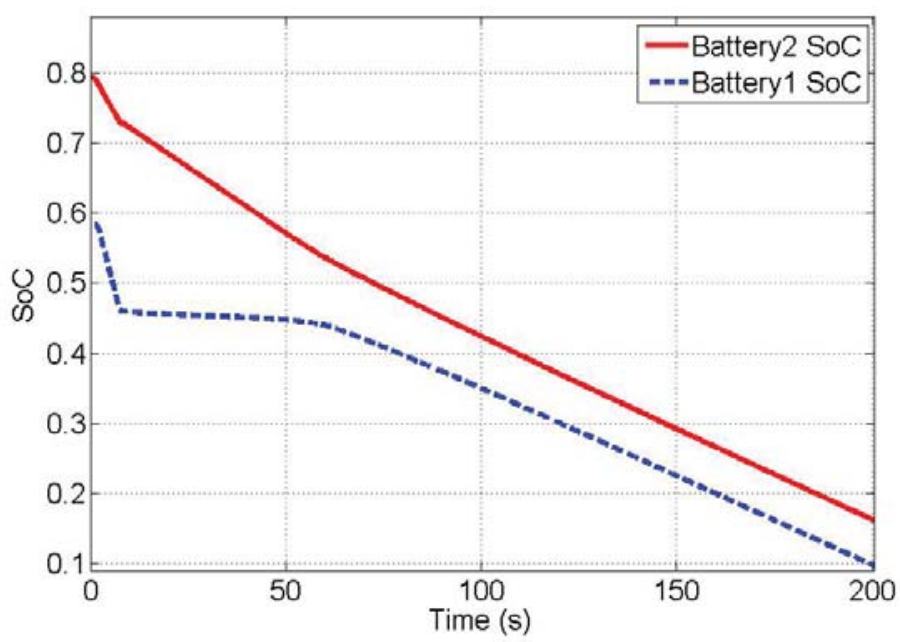

Figure 39: Scenario II: SoC zoomed in image, the gap between SoCs after $t=100 \mathrm{~s}$ is around 0.1 , validated the estimation

charging/discharging coefficients were performed as well. But the unstable or heavy load scenarios have not been tested, besides the battery model used in this experiment was an ideal constant voltage source. The drop voltage in battery might affect the SoC balancing but not this is included in this study. 


\section{Conclusion and future work}

This thesis presented a SoC balancing load sharing method. It is a modified droop control method. Its working principle, benefits and limitations were demonstrated and analyzed, and several working condition simulations were performed. Generally it modifies the droop equation based on its sensed SoC states; more precisely, the droop control reference voltage based on battery SoC value. Mathematical analysis showed this method had limits for choosing its SoC droop factor, and there is a trade off between SoC balancing speed and current sharing. As a result, all battery units applying this method in a micro system approach an identical SoC value. The bus voltage becomes related to the average SoC status in system. This feature, for a system with multiple storage devices, could be helpful in keeping a steady system and retaining sufficient storage capacity, load planning and shedding. A hardware test, using DSP and DC micro systems, was conducted to validate this method. Both simulation and hardware results showed that this method could handle the SoC balancing problem well and could put the SoC information on the system bus. This approach gives all devices in this system access to the overall battery SoC information by measuring the bus voltage without special communication lines. Such feature could be useful for load planning and shedding in distributed power supply systems.

Several models used in simulation and hardware test in this thesis, including battery and load model, were ideal or simplified. The battery's nonlinear V-SoC relation was not considered in the hardware test, and the charging/discharging feature of the battery was not applied in both the simulation and hard ware test models either.

The future work would be digging deeper into the battery charging/discharging 
coefficient compensation study, hardware test on real batteries and considering both charging and discharging scenarios. The SoC droop factor $\alpha$ needs more study too, because its value affects the balancing effect and battery operation as well, which makes it a good topic for optimization study. For real application, the battery discharging coefficient is not constant, its changing in could affect the balancing result. Lastly, the voltage-SoC detection needs more improvement, both in real voltage signal sampling and processing. 


\section{References}

\section{References}

[1] Z. Moussaoui, I. Batarseh, H. Lee, and C. Kennedy. An overview of the control scheme for distributed power systems. In Southcon/96, pages 584591, Jun 1996.

[2] I. Batarseh, K. Siri, and H. Lee. Investigation of the output droop characteristics of parallel-connnected dc-dc converters. In Power Electronics Specialists Conference, pages 1342-1351 vol.2, Jun 1994.

[3] H.R. Chamorro and G. Ramos. Microgrid central fuzzy controller for active and reactive power flow using instantaneous power measurements. In IEEE Power and Energy Conference at Illinois, pages 1-6, Feb 2011.

[4] F. Luo, Y.M. Lai, K.H. Loo, C.K. Tse, and Xinbo Ruan. A generalized droop-control scheme for decentralized control of inverter-interfaced microgrids. In IEEE International Symposium on Circuits and Systems, pages 1320-1323, May 2013.

[5] A. Maknouninejad, Zhihua Qu, F.L. Lewis, and A. Davoudi. Optimal, nonlinear, and distributed designs of droop controls for dc microgrids. IEEE Transactions on Smart Grid, 5(5):2508-2516, Sept 2014.

[6] H. Bevrani and S. Shokoohi. An intelligent droop control for simultaneous voltage and frequency regulation in islanded microgrids. IEEE Transactions on Smart Grid, 4(3):1505-1513, Sept 2013.

[7] A. Khaligh and Zhihao Li. Battery, ultracapacitor, fuel cell, and hybrid energy storage systems for electric, hybrid electric, fuel cell, and plug-in 
hybrid electric vehicles: State of the art. IEEE Transactions on Vehicular Technology, 59(6):2806-2814, July 2010.

[8] D. Linden and T.B. Reddy. Handbook of batteries. McGraw-Hill handbooks. McGraw-Hill, 2002.

[9] Huijun Li, Chenglin Liao, and Lifang Wang. Research on state-of-charge estimation of battery pack used on hybrid electric vehicle. In Asia-Pacific Power and Energy Engineering Conference., pages 1-4, March 2009.

[10] K. Kandasamy, D.M. Vilathgamuwa, and G. Foo. Inter-module soc balancing control for chb based bess using multi-dimensional modulation. In Industrial Technology (ICIT), pages 1630-1635, Feb 2013.

[11] P. Chanhom, S. Sirisukprasert, and N. Hatti. Dc-link voltage optimization for soc balancing control of a battery energy storage system based on a 7level cascaded pwm converter. In Electrical Engineering/Electronics, Computer, Telecommunications and Information Technology, pages 1-4, May 2012.

[12] Karthik Kandasamy, D.M. Vilathgamuwa, and King-Jet Tseng. Double star chopper cell converter for battery electric vehicles with inter-module soc balancing and fault tolerant control. In Industrial Electronics Society, pages 2991-2996, Oct 2014.

[13] J.M. Guerrero, Poh Chiang Loh, Tzung-Lin Lee, and M. Chandorkar. Advanced control architectures for intelligent microgrids x2014;part ii: Power quality, energy storage, and ac/dc microgrids. IEEE Transactions on Industrial Electronics, 60(4):1263-1270, April 2013. 
[14] L. Maharjan, S. Inoue, H. Akagi, and J. Asakura. State-of-charge (soc)balancing control of a battery energy storage system based on a cascade pwm converter. IEEE Transactions on Power Electronics, 24(6):1628-1636, June 2009 .

[15] Haihua Zhou, T. Bhattacharya, Duong Tran, T.S.T. Siew, and A.M. Khambadkone. Composite energy storage system involving battery and ultracapacitor with dynamic energy management in microgrid applications. IEEE Transactions on Power Electronics, 26(3):923-930, March 2011.

[16] Shiguo Luo, Zhihong Ye, Ray-Lee Lin, and F.C. Lee. A classification and evaluation of paralleling methods for power supply modules. In 30th Annual IEEE Power Electronics Specialists Conference., volume 2, pages 901-908 vol.2, 1999.

[17] Xiaonan Lu, Kai Sun, J.M. Guerrero, J.C. Vasquez, and Lipei Huang. Stateof-charge balance using adaptive droop control for distributed energy storage systems in dc microgrid applications. IEEE Transactions on Industrial Electronics, 61(6):2804-2815, June 2014.

[18] Xiaonan Lu, Kai Sun, J.M. Guerrero, J.C. Vasquez, Lipei Huang, and R. Teodorescu. Soc-based droop method for distributed energy storage in dc microgrid applications. In Industrial Electronics, pages 1640-1645, May 2012.

[19] Norman S. Nise. Control Systems Engineering. John Wiley \& Sons, Inc., New York, NY, USA, 3rd edition, 2000.

[20] T.R. Crompton. Battery Reference Book. Electronics \& Electrical. Newnes, 2000. 
[21] Ieee standard preferred voltage ratings for alternating-current electrical systems and equipment operating at voltages above $230 \mathrm{kv}$ nominal. IEEE Std 1312-1993, pages i-2, 1993.

[22] Ieee guide for voltage regulation and reactive power compensation at 1000 kv ac and above. IEEE Std 1860-2014, pages 1-41, July 2014.

[23] Ieee standard for rotating electric machinery for rail and road vehicles. IEEE Std 11-2000, pages i-, 2000.

[24] Ieee guide for the protection of stationary battery systems. IEEE Std 13751998, pages i-, 1998.

[25] A. Khaligh and Zhihao Li. Battery, ultracapacitor, fuel cell, and hybrid energy storage systems for electric, hybrid electric, fuel cell, and plug-in hybrid electric vehicles: State of the art. IEEE Transactions on Vehicular Technology, 59(6):2806-2814, July 2010.

[26] P.F. Ribeiro, B.K. Johnson, M.L. Crow, A. Arsoy, and Y. Liu. Energy storage systems for advanced power applications. Proceedings of the IEEE, 89(12):1744-1756, Dec 2001.

[27] H.A. Melo and C. Heinrich. Energy balance in a renewable energy community. In Environment and Electrical Engineering, pages 1-4, May 2011.

[28] Yixin Zhu, Fang Zhuo, and Liansong Xiong. Communication platform for energy management system in a master-slave control structure microgrid. In Power Electronics and Motion Control Conference, volume 1, pages 141145, June 2012. 
[29] Shu-Hung Liao, Jen-Hao Teng, Yung-Ching Huang, and Dong-Jing Lee. Optimal energy storage system planning for microgrids with contract capacity constraint. In Power Electronics Conference, pages 1452-1457, May 2014.

[30] Huang Youwei, Zhang Xu, He Junping, and Qin Yi. The improvement of micro grid hybrid energy storage system operation mode. In Transmission and Distribution Conference and Exposition, pages 1-6, April 2014. 


\section{$9 \quad$ Appendix}

9.1 Simulation block scheme in MATLAB/Sinmulink
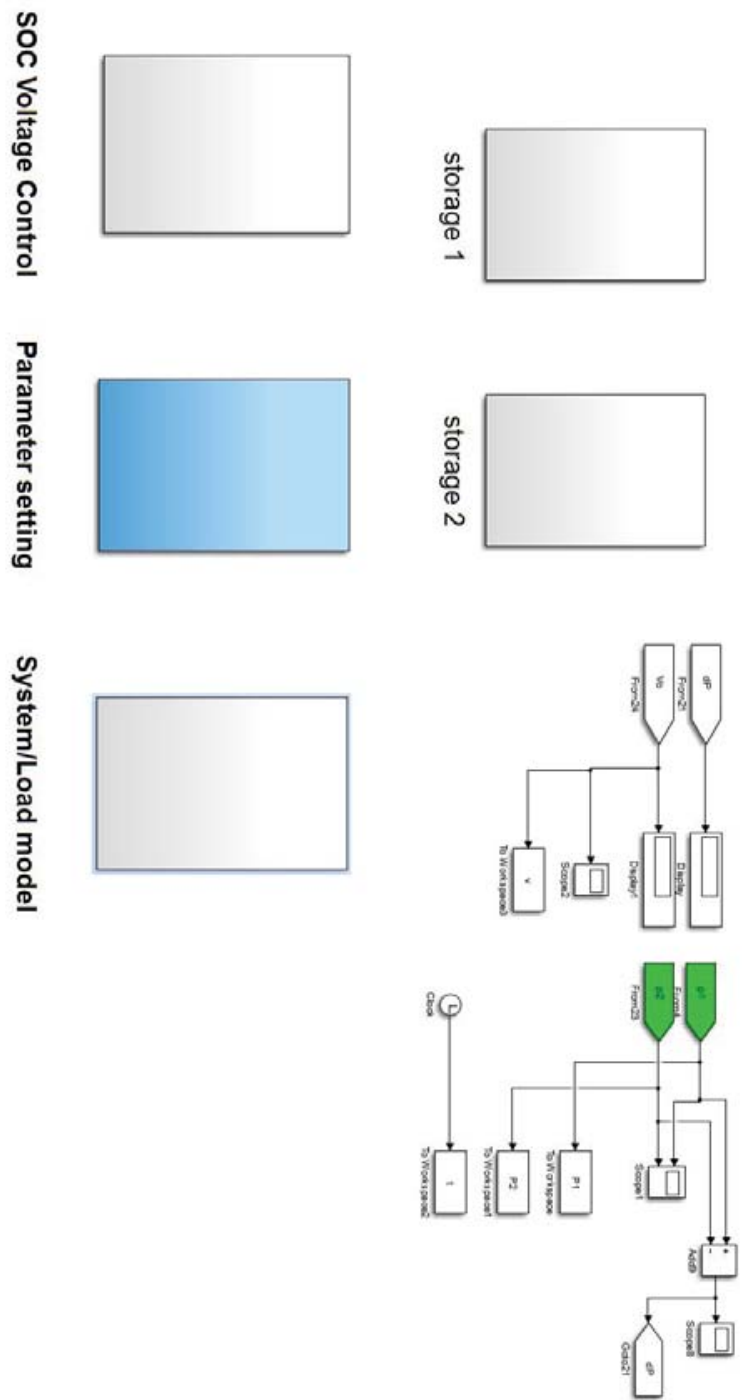

Figure 40: Main structure of simulation model 


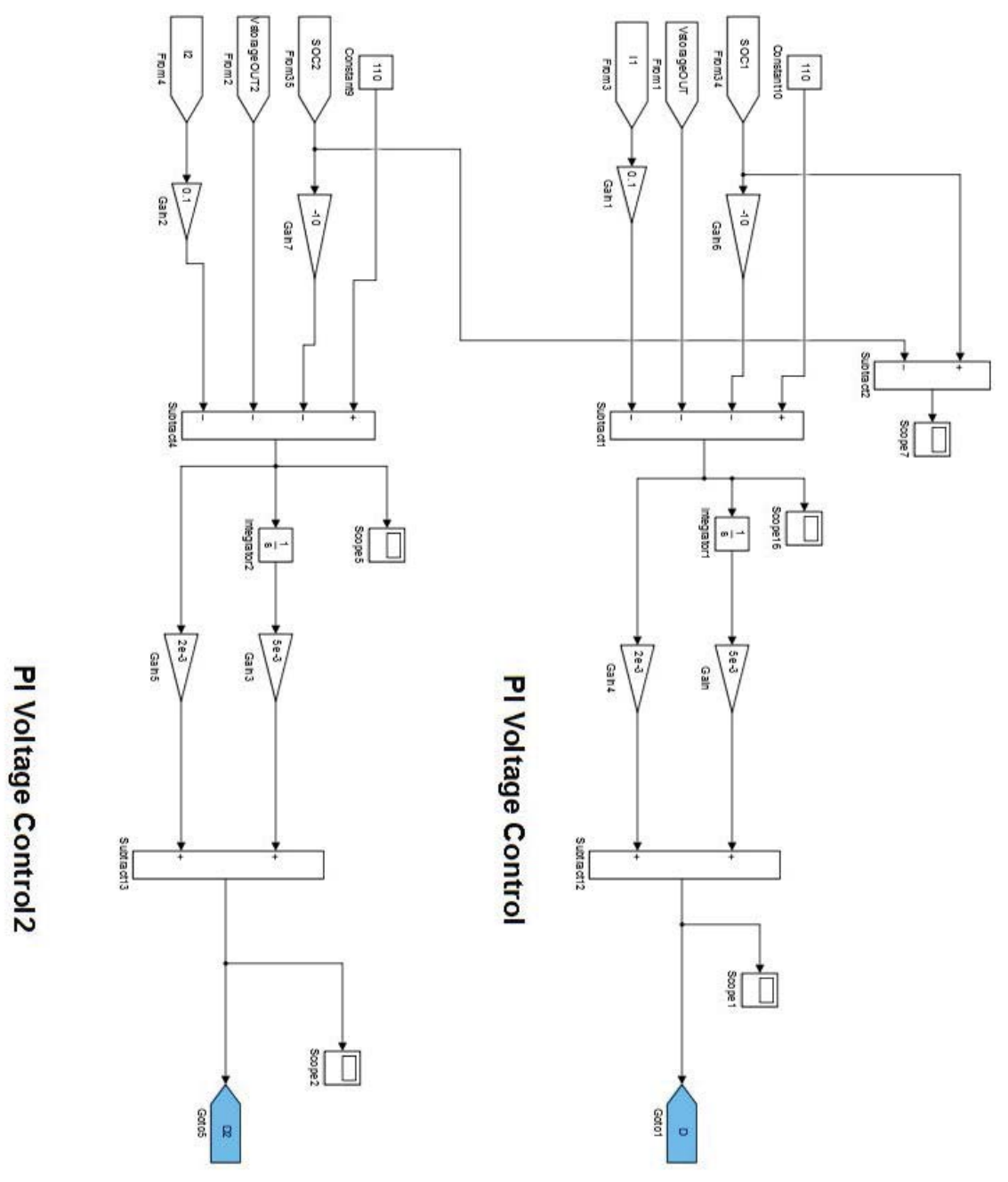

Figure 41: Control part applying SoC balancing control 


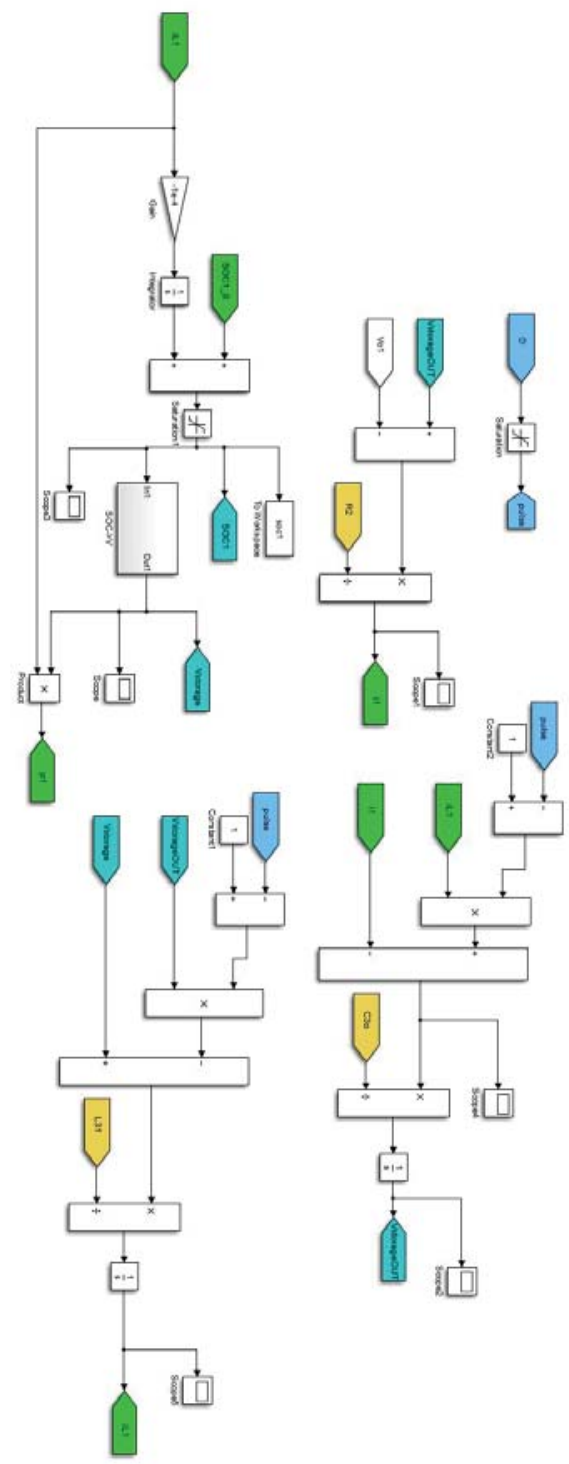

Figure 42: Battery model with converter built for simulation 


\subsection{Control model for electronic drive board in Sinmulink}

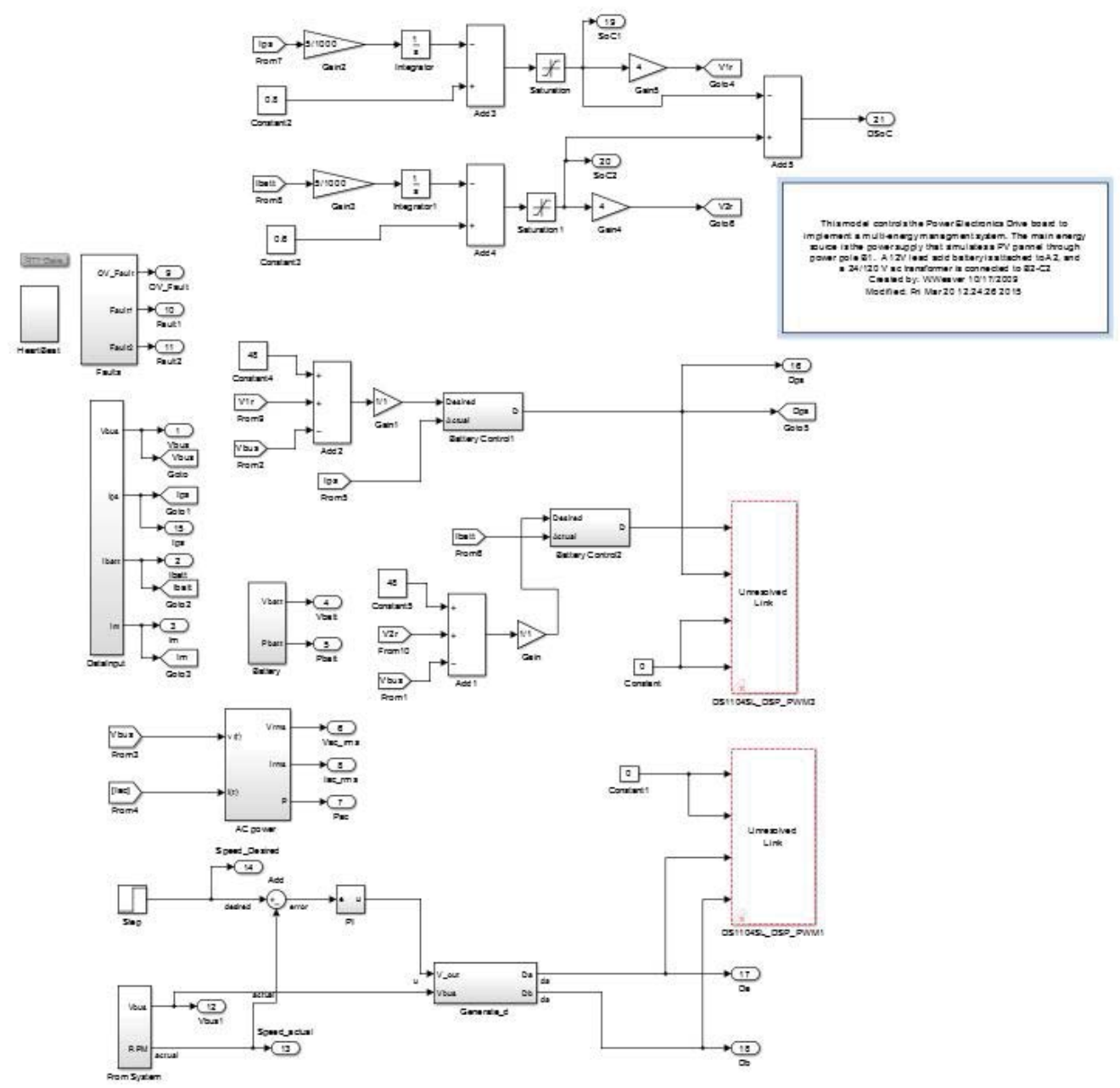

Figure 43: control model applying SoC balancing control 
9.3 Hardware test material

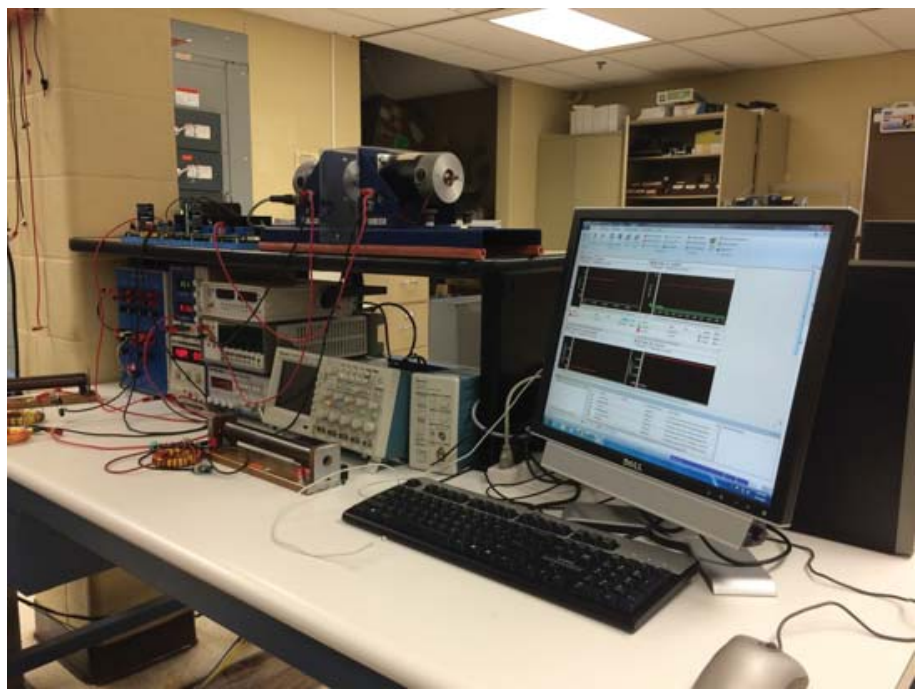

Figure 44: Experiment devices shot 


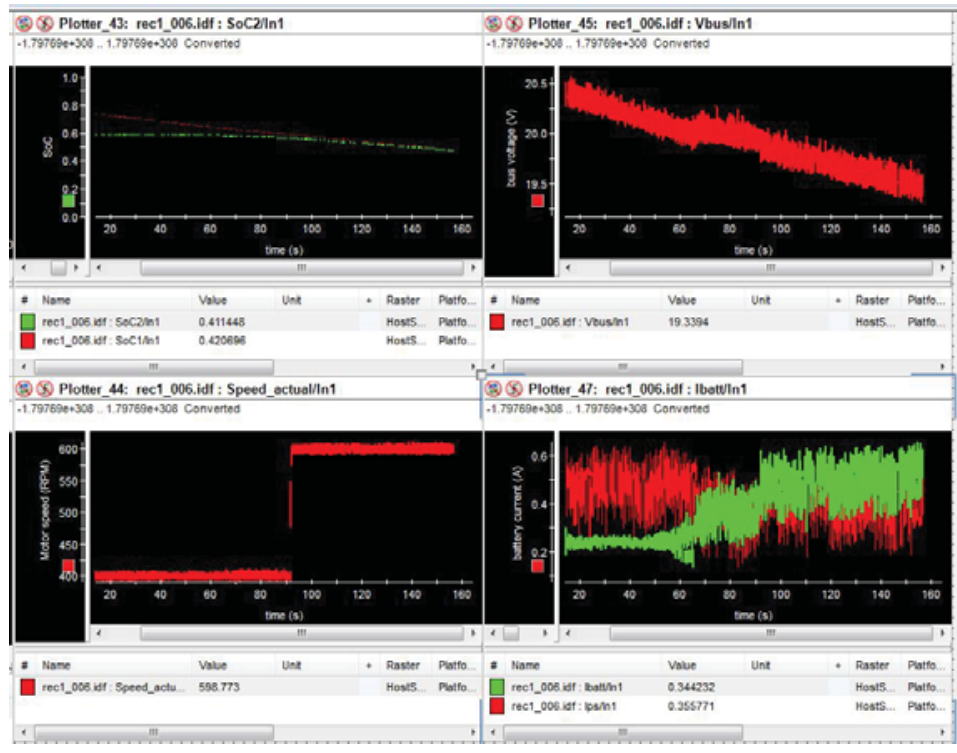

Figure 45: Original data gathered in Dspace control desk 


\begin{tabular}{|c|c|c|c|c|}
\hline Smple point(s) & Bttery 1 curent(A) & Bttery2 curent(A) & Motor Speed(RPM) & Bus voltage(V) \\
\hline 0 & $0.00 E+00$ & $4.86 \mathrm{E}-02$ & & 0.510125117 \\
\hline $9.98 \mathrm{E}-05$ & $9.98 \mathrm{E}-05$ & 0.048585571 & & 0.510128846 \\
\hline 0.000199973 & 0.000199973 & 0.04849034 & & 0.51010141 \\
\hline 0.000299804 & 0.000299804 & 0.048493718 & & 0.509949737 \\
\hline 0.000399945 & 0.000399945 & 0.048497062 & & 0.510079732 \\
\hline 0.000499776 & 0.000499776 & 0.048500372 & & 0.51030181 \\
\hline 0.000599918 & 0.000599918 & 0.04850365 & & 0.510241517 \\
\hline 0.000699749 & 0.000699749 & 0.048506895 & & 0.510244082 \\
\hline 0.000799891 & 0.000799891 & 0.048510107 & & 0.510246622 \\
\hline 0.000899722 & 0.000899722 & 0.048513287 & & 0.510218008 \\
\hline 0.000999863 & 0.000999863 & 0.048516436 & & 0.510251937 \\
\hline 0.001099694 & 0.001099694 & 0.048519553 & & 0.510254398 \\
\hline 0.001199836 & 0.001199836 & 0.048522638 & & 0.510287962 \\
\hline 0.001299667 & 0.001299667 & 0.048525693 & & 0.510258935 \\
\hline 0.001399809 & 0.001399809 & 0.048528717 & & 0.510385838 \\
\hline 0.00149964 & 0.00149964 & 0.048531711 & & 0.510324704 \\
\hline 0.001599782 & 0.001599782 & 0.048534676 & & 0.510357565 \\
\hline 0.001699612 & 0.001699612 & 0.04853761 & & 0.510296714 \\
\hline 0.001799754 & 0.001799754 & 0.048540515 & & 0.510174216 \\
\hline 0.001899585 & 0.001899585 & 0.048543391 & & 0.510021815 \\
\hline 0.001999727 & 0.001999727 & 0.048546239 & & 0.509995449 \\
\hline 0.002099558 & 0.002099558 & 0.048549058 & & 0.510187243 \\
\hline 0.0021997 & 0.0021997 & 0.048551848 & & 0.510128095 \\
\hline 0.00229953 & 0.00229953 & 0.048554611 & & 0.510100666 \\
\hline 0.002399361 & 0.002399361 & 0.048557346 & & 0.510166896 \\
\hline 0.002499503 & 0.002499503 & 0.048560054 & & 0.510263591 \\
\hline 0.002599334 & 0.002599334 & 0.048562735 & & 0.510390448 \\
\hline 0.002699476 & 0.002699476 & 0.048565389 & & 0.510453779 \\
\hline 0.002799618 & 0.002799618 & 0.048568016 & & 0.510360838 \\
\hline 0.002899449 & 0.002899449 & 0.048570617 & & 0.51036221 \\
\hline 0.002999279 & 0.002999279 & 0.048573192 & & 0.510363569 \\
\hline 0.003099421 & 0.003099421 & 0.048575741 & & 0.510333786 \\
\hline 0.003199252 & 0.003199252 & 0.048578265 & & 0.510335428 \\
\hline 0.003299394 & 0.003299394 & 0.048580764 & & 0.510492694 \\
\hline 0.003399225 & 0.003399225 & 0.048583237 & & 0.510523876 \\
\hline 0.003499367 & 0.003499367 & 0.048585686 & & 0.510585873 \\
\hline 0.003599197 & 0.003599197 & 0.048588111 & & 0.510709507 \\
\hline 0.003699339 & 0.003699339 & 0.048590511 & & 0.510707392 \\
\hline 0.00379917 & 0.00379917 & 0.048592887 & & 0.510892066 \\
\hline 0.003899312 & 0.003899312 & 0.048595239 & & 0.510856998 \\
\hline 0.003999143 & 0.003999143 & 0.048597568 & & 0.510853408 \\
\hline 0.004099285 & 0.004099285 & 0.048599874 & & 0.510818727 \\
\hline 0.004199116 & 0.004199116 & 0.048602156 & & 0.510877776 \\
\hline 0.004299257 & 0.004299257 & 0.048604416 & & 0.510936235 \\
\hline 0.004399088 & 0.004399088 & 0.048606653 & & 0.510994109 \\
\hline 0.00449923 & 0.00449923 & 0.048608868 & & 0.511020276 \\
\hline
\end{tabular}

Figure 46: Experiment data1 collected using Dspace recorder(Incomplete) 


\begin{tabular}{|c|c|c|c|c|}
\hline 4.52687549 & 4.52687549 & 2.037272239 & 3.254420101 & 1.03712765 \\
\hline 4.526975632 & 4.526975632 & 2.037212016 & 3.2218759 & 1.036904078 \\
\hline 4.527075463 & 4.527075463 & 2.037250053 & 3.189657141 & 1.037709964 \\
\hline 4.527175605 & 4.527175605 & 2.037287708 & 3.157760569 & 1.037542825 \\
\hline 4.527275436 & 4.527275436 & 2.037324987 & 3.126182964 & 1.037470742 \\
\hline 4.527375578 & 4.527375578 & 2.037361894 & 3.094921134 & 1.037025844 \\
\hline 4.527475408 & 4.527475408 & 2.037398431 & 3.063971923 & 1.03621186 \\
\hline 4.52757555 & 4.52757555 & 2.037434603 & 3.033332203 & 1.035997446 \\
\hline 4.527675381 & 4.527675381 & 2.037470413 & 3.002998881 & 1.035878561 \\
\hline 4.527775523 & 4.527775523 & 2.037505865 & 2.972968893 & 1.035605224 \\
\hline 4.527875354 & 4.527875354 & 2.037540963 & 2.943239204 & 1.035303494 \\
\hline 4.527975496 & 4.527975496 & 2.03757571 & 2.913806812 & 1.03516042 \\
\hline 4.528075327 & 4.528075327 & 2.037610109 & 2.884668743 & 1.035143288 \\
\hline 4.528175468 & 4.528175468 & 2.037644164 & 2.855822056 & 1.035032944 \\
\hline 4.528275299 & 4.528275299 & 2.037677879 & 2.827263835 & 1.034643552 \\
\hline 4.528375441 & 4.528375441 & 2.037711256 & 2.798991197 & 1.034289182 \\
\hline 4.528475272 & 4.528475272 & 2.0377443 & 2.771001285 & 1.033938356 \\
\hline 4.528575414 & 4.528575414 & 2.037777013 & 2.743291272 & 1.033808933 \\
\hline 4.528675245 & 4.528675245 & 2.037809399 & 2.71585836 & 1.033680805 \\
\hline 4.528775387 & 4.528775387 & 2.037841461 & 2.688699776 & 1.033927493 \\
\hline 4.528875217 & 4.528875217 & 2.037873203 & 2.661812778 & 1.033549155 \\
\hline 4.528975359 & 4.528975359 & 2.037806971 & 2.63519465 & 1.033548136 \\
\hline 4.52907519 & 4.52907519 & 2.037839057 & 2.608842704 & 1.033298104 \\
\hline 4.529175021 & 4.529175021 & 2.037870823 & 2.582754277 & 1.033206212 \\
\hline 4.529275163 & 4.529275163 & 2.037804615 & 2.556926734 & 1.03323975 \\
\hline 4.529374994 & 4.529374994 & 2.037934381 & 2.531357467 & 1.034144536 \\
\hline 4.529475135 & 4.529475135 & 2.037965194 & 2.506043892 & 1.033608388 \\
\hline 4.529575277 & 4.529575277 & 2.037995698 & 2.480983453 & 1.033139858 \\
\hline 4.529675108 & 4.529675108 & 2.038025897 & 2.456173619 & 1.032862781 \\
\hline 4.52977525 & 4.52977525 & 2.038055795 & 2.431611882 & 1.03280637 \\
\hline 4.529875081 & 4.529875081 & 2.038085393 & 2.407295764 & 1.032532627 \\
\hline 4.529974912 & 4.529974912 & 2.038114695 & 2.383222806 & 1.032043727 \\
\hline 4.530075054 & 4.530075054 & 2.038143704 & 2.359390578 & 1.031466331 \\
\hline 4.530174884 & 4.530174884 & 2.038172424 & 2.335796672 & 1.030863582 \\
\hline 4.530275026 & 4.530275026 & 2.038200856 & 2.312438705 & 1.030547012 \\
\hline 4.530374857 & 4.530374857 & 2.038229003 & 2.289314318 & 1.030140223 \\
\hline 4.530474999 & 4.530474999 & 2.03825687 & 2.266421175 & 1.029862014 \\
\hline 4.53057483 & 4.53057483 & 2.038284457 & 2.243756963 & 1.029679971 \\
\hline 4.530674972 & 4.530674972 & 2.038311769 & 2.221319394 & 1.029344109 \\
\hline 4.530774802 & 4.530774802 & 2.038338807 & 2.1991062 & 1.028855966 \\
\hline 4.530874944 & 4.530874944 & 2.038365576 & 2.177115138 & 1.028746239 \\
\hline 4.530974775 & 4.530974775 & 2.038392076 & 2.155343986 & 1.02938468 \\
\hline 4.531074917 & 4.531074917 & 2.038418312 & 2.133790547 & 1.029580946 \\
\hline 4.531174748 & 4.531174748 & 2.038444285 & 2.112452641 & 1.029214946 \\
\hline 4.53127489 & 4.53127489 & 2.038469998 & 2.091328115 & 1.028852606 \\
\hline 4.531374721 & 4.531374721 & 2.038495454 & 2.070414834 & 1.028400506 \\
\hline 4.531474862 & 4.531474862 & 2.038520656 & 2.049710685 & 1.028108566 \\
\hline
\end{tabular}

Figure 47: Experiment data2 collected using Dspace recorder(Incomplete) 